\title{
Phenomena of Blowup and Global Existence of the Solution to a Nonlinear Schrödinger Equation
}

\author{
Xiaowei An, ${ }^{1,2}$ Desheng Li, ${ }^{3}$ and Xianfa Song ${ }^{3}$ \\ ${ }^{1}$ School of Electrical Engineering and Automation, Tianjin University, Tianjin 300072, China \\ ${ }^{2}$ Department of Basic Curriculum, The Chinese People's Armed Police Force Academy, Lang Fang, He Bei 065000, China \\ ${ }^{3}$ Department of Mathematics, School of Science, Tianjin University, Tianjin 300072, China
}

Correspondence should be addressed to Xianfa Song; songxianfa@tju.edu.cn

Received 26 August 2013; Accepted 13 October 2013

Academic Editor: Sining Zheng

Copyright (C) 2013 Xiaowei An et al. This is an open access article distributed under the Creative Commons Attribution License, which permits unrestricted use, distribution, and reproduction in any medium, provided the original work is properly cited.

We consider the following Cauchy problem: $-i u_{t}=\Delta u-V(x) u+f\left(x,|u|^{2}\right) u+\left(W(x) \star|u|^{2}\right) u, x \in \mathbb{R}^{N}, t>0, u(x, 0)=u_{0}(x), x \in \mathbb{R}^{N}$, where $V(x)$ and $W(x)$ are real-valued potentials and $V(x) \geq 0$ and $W(x)$ is even, $f\left(x,|u|^{2}\right)$ is measurable in $x$ and continuous in $|u|^{2}$, and $u_{0}(x)$ is a complex-valued function of $x$. We obtain some sufficient conditions and establish two sharp thresholds for the blowup and global existence of the solution to the problem.

\section{Introduction}

In this paper, we consider the following Cauchy problem:

$$
\begin{aligned}
-i u_{t}= & \Delta u-V(x) u+f\left(x,|u|^{2}\right) u \\
& +\left(W(x) \star|u|^{2}\right) u, \quad x \in \mathbb{R}^{N}, t>0, \\
& u(x, 0)=u_{0}(x) \in \Sigma, \quad x \in \mathbb{R}^{N},
\end{aligned}
$$

where $V(x)$ and $W(x)$ are real-valued potentials, $V(x) \geq$ 0 and $W(x)$ is even, $f\left(x,|u|^{2}\right)$ is measurable in $x$ and continuous in $|u|^{2}$,

$$
\begin{aligned}
& \left(W(x) \star|u|^{2}\right) u(x) \\
& \quad=\left(\int_{\mathbb{R}^{N}} W(x-y)|u(y)|^{2} d y\right) u(x),
\end{aligned}
$$

$u_{0}(x)$ is a complex-valued function of $x$, and $\Sigma$ is the Hilbert space:

$$
\Sigma=\left\{u \in H^{1}\left(\mathbb{R}^{N}\right), \int_{\mathbb{R}^{N}} V(x)|u|^{2} d x<+\infty\right\},
$$

with the inner product

$$
\langle\varphi, \psi\rangle=\int_{\mathbb{R}^{N}}[\varphi \bar{\psi}+\nabla \varphi \cdot \nabla \bar{\psi}+V(x) \varphi \bar{\psi}] d x
$$

and the norm

$$
\|u\|_{\Sigma}^{2}=\int_{\mathbb{R}^{N}}\left(|u|^{2}+|\nabla u|^{2}+V(x)|u|^{2}\right) d x .
$$

Model (1) appears in the theory of Bose-Einstein condensation, nonlinear optics and theory of water waves (see $[1,2])$.

For convenience, denote $1 /(N-2)^{+}=+\infty$ when $N=$ 1,2 and $(N-2)^{+}=N-2$ when $N \geq 3$. We also give some assumptions on $V(x), f(x, s)$, and $W(x)$ as follows.

(V1) $V(x) \geq 0$ and $V(x) \in L^{r}\left(\mathbb{R}^{N}\right)+L^{\infty}\left(\mathbb{R}^{N}\right)$ for $r \geq 1$, $r>N / 2$.

(V2) $V(x) \geq 0, V(x) \in \mathbf{S}_{1}^{c}$, and $\left|D^{\alpha} V\right|$ is bounded for all $|\alpha| \geq 2$. Here $\boldsymbol{S}_{1}^{c}$ is the complementary set of $\mathbf{S}_{1}=$ $\{V(x)$ satisfies $(V 1)\}$.

(f1) $f(x, s): \mathbb{R}^{N} \times \mathbb{R} \rightarrow \mathbb{R}$ is measurable in $x$ and continuous in $|u|^{2}$ with $f(x, 0)=0$. 
Assume that, for every $k>0$, there exists $L(k)<+\infty$ such that $\left|f\left(x, s_{1}\right)-f\left(x, s_{2}\right)\right| \leq L(k)\left|s_{1}-s_{2}\right|$ for all $0 \leq s_{1}<s_{2}<k$. Here

$$
\begin{gathered}
L(k) \in C([0, \infty)), \quad \text { if } N=1, \\
L(k) \leq C\left(1+k^{\alpha}\right) \text { with } 0 \leq \alpha<\frac{2}{(N-2)^{+}}, \text {if } N \geq 2 .
\end{gathered}
$$

(W1) $W(x)$ is even and $W(x) \in L^{q}\left(\mathbb{R}^{N}\right)+L^{\infty}\left(\mathbb{R}^{N}\right)$ for some $q \geq 1, q>N / 4$.

First, we consider the local well-posedness of (1). We have a proposition as follows.

Proposition 1 (local existence result). Assume that $(f 1)$ and $(W 1)$ are true, $V(x)$ satisfies $(V 1)$ or $(V 2)$, and $u_{0} \in \Sigma$. Then there exists a unique solution $u$ of (1) on a maximal time interval $\left[0, T_{\max }\right)$ such that $u \in C\left(\Sigma ;\left[0, T_{\max }\right)\right)$ and either $T_{\max }=+\infty$ or else

$$
T_{\max }<+\infty, \quad \lim _{t \rightarrow T_{\max }}\|u(\cdot, t)\|_{\Sigma}=+\infty .
$$

Definition 2. If $u \in C(\Sigma ;[0, T))$ with $T=\infty$, we say that the solution $u$ of (1) exists globally. If $u \in C(\Sigma ;[0, T))$ with $T<$ $+\infty$ and $\lim _{t \rightarrow T}\|u(\cdot, t)\|_{\Sigma} \rightarrow+\infty$, we say that the solution $u$ of (1) blows up in finite time.

This paper is directly motivated by $[1,3-5]$. Since Cazevave established some results on blowup and global existence of the solutions to (1) with (V1), (f1), and (W1) in [1], we are interested in the problems such as "What are the results about the blowup and global existence of the solutions to (1) with (V2), (f1), and (W1)?" On the other hand, since Gan et al. had established some sharp thresholds for global existence and blowup of the solution to the related problems to (1) (see [35] and the references therein), it is a natural way to consider the sharp threshold for global existence and blowup of the solution to (1).

About the topic of global existence and blowup in finite time, there are many results on the special cases of (1). We will recall some results on the following Cauchy problem:

$$
\begin{gathered}
-i u_{t}=\Delta u+f\left(|u|^{2}\right) u, \quad x \in \mathbb{R}^{N}, t>0, \\
u(x, 0)=u_{0}(x), \quad x \in \mathbb{R}^{N} .
\end{gathered}
$$

In [6], Glassey established some blowup results for (8). In [7], Berestyki and Cazenave established the sharp threshold for blowup of (8) with supercritical nonlinearity by considering a constrained variational problem. In [8], Weinstein presented a relationship between the sharp criterion for the global solution of (8) and the best constant in the Gagliardo-Nirenberg inequality. In [9], Cazenave and Weisseler established the local existence and uniqueness of the solution to (8) with $f\left(|u|^{2}\right) u=|u|^{4 / N} u$. Very recently, Tao et al. in [10] studied the Cauchy problem (8) with $f\left(|u|^{2}\right) u=\mu|u|^{p_{1}} u+v|u|^{p_{2}} u$, where $\mu$ and $\nu$ are real numbers, $0<p_{1}<p_{2}<4 /(N-2)$ with $N \geq 3$, and established the results on local and global wellposedness, asymptotic behavior (scattering), and finite time blowup under some assumptions. Other sharp thresholds were established by Chen et al. in $[11,12]$. The following Cauchy problem

$$
\begin{aligned}
-i u_{t}= & \frac{1}{2} \Delta u-|x|^{2} u+K(x)|u|^{p} u \\
& +Q(x)|u|^{q} u, \quad x \in \mathbb{R}^{N}, t>0, \\
u(x, 0)=u_{0}(x), \quad x \in \mathbb{R}^{N} &
\end{aligned}
$$

is also a special case of (1), where $0<p<q<4 /(N-2)$ with $N \geq 3$. In [2], Oh obtained the local well-posedness and global existence results of (9) under some conditions. In $[3,5]$, Gan et al. and Zhang, respectively, established the sharp thresholds for the global existence and blowup of the solutions to (9) under some conditions. In [4], Gan et al. dealt with

$$
\begin{gathered}
-i u_{t}=\frac{1}{2} \Delta u+a|u|^{p} u+E_{1}\left(|u|^{2}\right) u, \\
x \in \mathbb{R}^{N}, t>0, \\
u(x, 0)=u_{0}(x), \quad x \in \mathbb{R}^{N},
\end{gathered}
$$

with $E_{1}(\xi)$ a singular integral operator, where $0<p<$ $4 /(N-2)$ with $N \geq 3$. They got the sharp threshold for global existence and blowup of the solution to (10) and the instability of the wave solutions. Very recently, Miao et al. also obtained some results on the blowup and global existence of the solution to a Hartree equation (see [13-15]). Naturally, we want to establish some new sharp thresholds for global existence and blowup of the solution to (1) in this paper and generalize these results above. Although the methods of our paper are inspired by the references above, our results, which will be stated in Section 2, are new and cover theirs.

This paper is organized as follows. In Section 2, we will recall some results of [1] and state our main results; then we will prove Proposition 1 and give some other properties. In Section 3, we will prove Theorems 3 and 4. In Section 4, we establish the sharp threshold for (1) with $V(x) \equiv 0$. In Section 5, we will prove Theorem 7.

\section{Our Main Results}

Now we will introduce some notations. Denote

$$
\begin{gathered}
F\left(x,|u|^{2}\right)=\int_{0}^{|u|^{2}} f(x, s) d s, \\
G\left(|u|^{2}\right)=\frac{1}{4} \int_{\mathbb{R}^{N}}\left(W(x) \star|u|^{2}\right)|u|^{2} d x, \\
h(u)=-V(x) u+f\left(x,|u|^{2}\right) u+\left(W(x) \star|u|^{2}\right) u, \\
H(u)=-\frac{1}{2} \int_{\mathbb{R}^{N}} V(x)|u|^{2} d x+\frac{1}{2} \int_{\mathbb{R}^{N}} F\left(x,|u|^{2}\right) d x \\
+\frac{1}{4} \int_{\mathbb{R}^{N}}\left(W(x) \star|u|^{2}\right)|u|^{2} d x,
\end{gathered}
$$


$\operatorname{mass}\left(L^{2}\right.$ norm $)$

$$
M(u):=\left(\int_{\mathbb{R}^{N}}|u(x, t)|^{2} d x\right)^{1 / 2},
$$

energy

$$
\begin{aligned}
E(u):= & \frac{1}{2} \int_{\mathbb{R}^{N}}\left(|\nabla u|^{2}+V(x)|u|^{2}\right) d x \\
& -\frac{1}{2} \int_{\mathbb{R}^{N}} F\left(x,|u|^{2}\right) d x \\
& -\frac{1}{4} \int_{\mathbb{R}^{N}}\left(W(x) \star|u|^{2}\right)|u|^{2} d x .
\end{aligned}
$$

In [1], Cazenave obtained some sufficient conditions on blowup and global existence of the solution to (1) with (V1), (f1), and (W1). The following two theorems can be looked at as the parallel results to Corollary 6.1.2 and Theorem 6.5.4 of [1], respectively.

Theorem 3 (global existence). Assume that $u_{0} \in \Sigma$, (V2) and $(f 1)$ are true, and

$$
W^{+}(x) \in L^{q}\left(\mathbb{R}^{N}\right)+L^{\infty}\left(\mathbb{R}^{N}\right)
$$

for some $q \geq 1, q \geq N / 2$ (and $q>1$ if $N=2$ ). Here $W^{+}=$ $\max (W(x), 0)$. Suppose further that there exist constants $c_{1}$ and $c_{2}$ such that $F\left(x,|u|^{2}\right) \leq c_{1}|u|^{2}+c_{2}|u|^{2 p+2}$ with $0<p<2 / N$. Then the solution of (1) exists globally. That is,

$$
\|u(\cdot, t)\|_{\Sigma}<+\infty \quad \forall 0<t<+\infty .
$$

Theorem 4 (blowup in finite time). Assume that $u_{0} \in \Sigma$ and $|x| u_{0} \in L^{2}\left(\mathbb{R}^{N}\right),(V 2),(f 1)$, and (W1) are true. Suppose further that

$$
\begin{gathered}
(N+2) F\left(x,|u|^{2}\right)-N|u|^{2} f\left(x,|u|^{2}\right) \leq 0, \\
2 V(x)+(x \cdot \nabla V(x)) \geq 0 \quad \text { a.e. } \\
2 W(x)+(x \cdot \nabla W(x)) \leq 0 \quad \text { a.e. }
\end{gathered}
$$

If (1) $E\left(u_{0}\right)<0$ or (2) $E\left(u_{0}\right)=0$ and $\mathfrak{I} \int_{\mathbb{R}^{N}}\left(x \cdot \nabla u_{0}\right) \bar{u}_{0} d x<0$, then the solution of (1) will blow up in finite time. That is, there exists $T_{\max }<\infty$ such that

$$
\lim _{t \rightarrow T_{\max }}\|u(\cdot, t)\|_{\Sigma}=\infty
$$

Denote

$$
\begin{aligned}
Q(u):= & 2 \int_{\mathbb{R}^{N}}|\nabla u|^{2} d x-\int_{\mathbb{R}^{N}}(x \cdot \nabla V(x))|u|^{2} d x \\
& +N \int_{\mathbb{R}^{N}}\left[F\left(x,|u|^{2}\right)-|u|^{2} f\left(x,|u|^{2}\right)\right] d x \\
& +\frac{1}{2} \int_{\mathbb{R}^{N}}\left((x \cdot \nabla W(x)) \star|u|^{2}\right)|u|^{2} d x .
\end{aligned}
$$

We will establish the first type of sharp threshold as follows.
Theorem 5 (sharp threshold I). Assume that $V(x) \equiv 0$ and $W(x) \in L^{q}\left(\mathbb{R}^{N}\right)$ with $N / 4<q<N / 2$. Suppose further that $f(x, 0)=0$ and there exist constants $c_{1}, c_{2}, c_{3}>0$ and $2 / N<$ $p_{1}, p_{2}, l<2 /(N-2)^{+}$such that

$$
\begin{gathered}
l F\left(x,|u|^{2}\right) \leq|u|^{2} f\left(x,|u|^{2}\right)-F\left(x,|u|^{2}\right) \\
\leq c_{1}|u|^{2 p_{1}+2}+c_{2}|u|^{2 p_{2}+2}, \\
\operatorname{NlW}(x)+(x \cdot \nabla W(x)) \leq 0 \leq c_{3} W(x)+(x \cdot \nabla W(x)) .
\end{gathered}
$$

Let $\omega$ be a positive constant satisfying

$$
d_{I}:=\inf _{\{u \in \Sigma \backslash\{0\} ; Q(u)=0\}}\left(\omega\|u\|_{2}^{2}+E(u)\right)>0,
$$

where $Q(u)$ is defined by (22). Suppose that $u_{0} \in H^{1}\left(\mathbb{R}^{N}\right)$ satisfies

$$
\omega\left\|u_{0}\right\|_{2}^{2}+E\left(u_{0}\right)<d_{I}
$$

Then

(1) if $Q\left(u_{0}\right)>0$, the solution of (1) exists globally;

(2) if $Q\left(u_{0}\right)<0,|x| u_{0} \in L^{2}\left(\mathbb{R}^{N}\right)$, and $\mathfrak{I} \int_{\mathbb{R}^{N}}(x$. $\left.\nabla u_{0}\right) \bar{u}_{0} d x<0$, the solution of (1) blows up in finite time.

Remark 6. Theorem 5 is only suitable for (1) with $V(x) \equiv 0$. To establish the sharp threshold for (1) with $V(x) \neq 0$, we will construct a type of cross-constrained variational problem and establish some cross-invariant manifolds. First, we introduce some functionals as follows:

$$
I_{\omega}(u)=\omega\|u\|_{2}^{2}+E(u),
$$

$$
\begin{gathered}
S_{\omega}(u)=2 \omega\|u\|_{2}^{2} \\
+\int_{\mathbb{R}^{N}}\left\{|\nabla u|^{2}+V(x)|u|^{2}-f\left(x,|u|^{2}\right)|u|^{2}\right. \\
\left.\quad-\left(W(x) \star|u|^{2}\right)|u|^{2}\right\} d x .
\end{gathered}
$$

Denote the Nehari manifold

$$
\mathcal{N}:=\left\{u \in \Sigma \backslash\{0\}, S_{\omega}(u)=0\right\}
$$

and cross-manifold

$$
\mathscr{C} \mathscr{M}:=\left\{u \in \Sigma \backslash\{0\}, S_{\omega}(u)<0, Q(u)=0\right\} .
$$

Define

$$
\begin{aligned}
& d_{\mathcal{N}}:=\inf _{\mathcal{N}} I_{\omega}(u), \\
& d_{\mathscr{M}}:=\inf _{\mathscr{C} \mathscr{M}} I_{\omega}(u), \\
& d_{I I}:=\min \left(d_{\mathscr{N}}, d_{\mathscr{M}}\right) .
\end{aligned}
$$

In Section 5, we will prove that $d_{I I}$ is always positive. Therefore, it is reasonable to define the following crossmanifold:

$$
\mathscr{K}:=\left\{u \in \Sigma \backslash\{0\}: I_{\omega}(u)<d_{I I}, S_{\omega}(u)<0, Q(u)<0\right\} .
$$

We give the second type of sharp threshold as follows. 
Theorem 7 (sharp threshold II). Assume that (f1), (W1), and (23). Suppose that

$$
W(x) \geq 0, \quad N l W(x)+(x \cdot \nabla W(x)) \leq 0
$$

and there exists a positive constant $c$ such that

$$
N l V(x)+(x \cdot \nabla V(x)) \geq c V(x) \geq 0
$$

with the same $l$ in (23). Assume further that the function $f\left(x,|u|^{2}\right)$ satisfies $f(x, 0)=0$ and

$$
\begin{gathered}
f\left(x,|u|^{2}\right) \leq f\left(x, k^{2}|u|^{2}\right), \quad f_{s}^{\prime}\left(x, k^{2}|u|^{2}\right) \leq f_{s}^{\prime}\left(x,|u|^{2}\right) \\
F\left(x, k^{2}|u|^{2}\right)-k^{2}|u|^{2} f\left(x, k^{2}|u|^{2}\right) \\
\leq k^{2}\left[F\left(x,|u|^{2}\right)-|u|^{2} f\left(x,|u|^{2}\right)\right]
\end{gathered}
$$

for $k>1$. Here $f_{s}^{\prime}(x, z)$ is the value of the partial derivative of $f(x, s)$ with respect to $s$ at the point $(x, z)$. If $u_{0} \in \Sigma$ and $|x| u_{0} \in L^{2}\left(\mathbb{R}^{N}\right)$ with $I_{\omega}\left(u_{0}\right)=\omega\left\|u_{0}\right\|_{2}^{2}+E\left(u_{0}\right)<d_{I I}$, then the solution of (1) blows up in finite time if and only if $u_{0} \in \mathscr{K}$.

Remark 8. (1) $f\left(x,|u|^{2}\right) \leq f\left(x, k^{2}|u|^{2}\right)$ implies that $k^{2} F\left(x,|u|^{2}\right) \leq F\left(x, k^{2}|u|^{2}\right)$ for $k>1$.

(2) The blowup of solution to (1) will benefit from the role of the potential $V$ if $V(x) \geq 0$. In some cases, the blowup of the solution to (1) can be delayed or prevented by the role of potential (see [16] and the references therein).

In the sequel, we use $C$ and $c$ to denote various finite constants; their exact values may vary from line to line.

First, we will give the proof of Proposition 1.

Proof of Proposition 1. If (V1) is true, then there exist $V_{1}(x) \in$ $L^{r}\left(\mathbb{R}^{N}\right)$ with $r \geq 1, r>N / 2$, and $V_{2}(x) \in L^{\infty}\left(\mathbb{R}^{N}\right)$ such that

$$
V(x)=V_{1}(x)+V_{2}(x) .
$$

Noticing that $0<2 r /(r-1)<2 N /(N-2)$, using Hölder's and Sobolev's inequalities, we have

$$
\begin{aligned}
\int_{\mathbb{R}^{N}} V(x)|u|^{2} d x= & \int_{\mathbb{R}^{N}} V_{1}(x)|u|^{2} d x+\int_{\mathbb{R}^{N}} V_{2}(x)|u|^{2} d x \\
\leq & \left(\int_{\mathbb{R}^{N}}\left|V_{1}(x)\right|^{r} d x\right)^{1 / r} \\
& \times\left(\int_{\mathbb{R}^{N}}|u|^{2 r /(r-1)} d x\right)^{(r-1) / r} \\
& +C \int_{\mathbb{R}^{N}}|u|^{2} d x \\
\leq & C \int_{\mathbb{R}^{N}}|\nabla u|^{2} d x+C \int_{\mathbb{R}^{N}}|u|^{2} d x
\end{aligned}
$$

for any $u \in H^{1}\left(\mathbb{R}^{N}\right)$. Consequently, we have

$$
\|u\|_{H^{1}} \leq\|u\|_{\Sigma} \leq C\|u\|_{H^{1}}
$$

By the results of Theorem 3.3.1 in [1], we have the local wellposedness result of (1) in $\Sigma$.

If (V2), (f1), and (W1) are true, similar to the proof of Theorem 3.5 in [2], we can establish the local well-posedness result of (1) in $\Sigma$. Roughly, we only need to replace $|u|^{p+1} u$ by $f\left(x,|u|^{2}\right) u+\left(W(x) \star|u|^{2}\right) u$ in the proof, and we can obtain similar results under the assumptions of (V2), (f1), and (W1).

Noticing that $\Im h(u) \bar{u}=0$ and $h(u)=H^{\prime}(u)$, following the method of [6] and the discussion in Chapter 3 of [1], one can obtain the conservation of mass and energy. We give the following proposition without proof.

Proposition 9. Assume that $u(x, t)$ is a solution of (1). Then

$$
\begin{aligned}
M(u) & =\left(\int_{\mathbb{R}^{N}}|u(x, t)|^{2} d x\right)^{1 / 2} \\
& =\left(\int_{\mathbb{R}^{N}}\left|u_{0}(x)\right|^{2} d x\right)^{1 / 2}=M\left(u_{0}\right), \\
E(u)= & \frac{1}{2} \int_{\mathbb{R}^{N}}\left\{|\nabla u|^{2}+V(x)|u|^{2}-F\left(x,|u|^{2}\right)\right\} d x \\
& -G\left(|u|^{2}\right)=E\left(u_{0}\right)
\end{aligned}
$$

for any $0 \leq t<T_{\max }$

We will recall some results on blowup and global existence of the solution to (1) with (V1), (f1), and (W1).

Theorem A (Corollary 6.12 of [1]). Assume that (V1), $(f 1)$, and (16). Suppose that there exist $A \geq 0$ and $0 \leq p<2 / N$ such that

$$
F\left(|u|^{2}\right) \leq A|u|^{2}\left(1+|u|^{2 p}\right)
$$

Then the maximal strong $H^{1}$-solution of (1) is global and $\sup \left\{\|u\|_{H^{1}}: t \in \mathbb{R}\right\}<\infty$ for every $u_{0} \in H^{1}\left(\mathbb{R}^{N}\right)$.

Theorem B (Theorem 6.54 of [1]). Assume that (V1), $(f 1)$, (W1), and (18)-(20). If $u_{0} \in H^{1}\left(\mathbb{R}^{N}\right),|x| u_{0} \in L^{2}\left(\mathbb{R}^{N}\right)$, and $E\left(u_{0}\right)<0$, then the $H^{1}$-solution of (1) will blow up in finite time.

Let $J(t)=\int_{\mathbb{R}^{N}}|x|^{2}|u|^{2} d x$. After some elementary computations, we obtain

$$
J^{\prime}(t)=4 \mathfrak{J} \int_{\mathbb{R}^{N}}(x \cdot \nabla u) \bar{u} d x, \quad J^{\prime \prime}(t)=4 Q(u) .
$$

We have the following proposition.

Proposition 10. Assume that $u(x, t)$ is a solution of (1) with $u_{0} \in \sum$ and $|x| u_{0} \in L^{2}\left(\mathbb{R}^{N}\right)$. Then the solution to (1) will blow up in finite time if either

(1) there exists a constant $c<0$ such that $J^{\prime \prime}(t)=4 Q(u) \leq$ $c<0$ or

(2) $J^{\prime \prime}(t)=4 Q(u) \leq 0$ and $J^{\prime}(0)=\Im \int_{\mathbb{R}^{N}}\left(x \cdot \nabla u_{0}\right) \bar{u}_{0} d x<$ 0 . 
Proof. Since $u_{0} \in \Sigma$ and $|x| u_{0} \in L^{2}\left(\mathbb{R}^{N}\right)$, we have

$$
\begin{aligned}
\left|J^{\prime}(0)\right| & <4 \int_{\mathbb{R}^{N}}\left|x \bar{u}_{0}\right|\left|\nabla u_{0}\right| d x \\
& \leq 8 \int_{\mathbb{R}^{N}}\left(\left|\nabla u_{0}\right|^{2}+\left|x u_{0}\right|^{2}\right) d x<+\infty
\end{aligned}
$$

(1) If $J^{\prime \prime}(t) \leq c<0$, integrating it from 0 to $t$, we get $J^{\prime}(t)<c t+J^{\prime}(0)$. Since $c<0$, we know that there exists a $t_{0} \geq \max \left(0, J^{\prime}(0) /-c\right)$ such that $J^{\prime}(t)<J^{\prime}\left(t_{0}\right)<0$ for $t>t_{0}$. On the other hand, we have

$$
0 \leq J(t)=J\left(t_{0}\right)+\int_{t_{0}}^{t} J^{\prime}(s) d s<J\left(t_{0}\right)+J^{\prime}\left(t_{0}\right)\left(t-t_{0}\right),
$$

which implies that there exists a $T_{\max }<+\infty$ satisfying

$$
\lim _{t \rightarrow T_{\max }} J(t)=0
$$

Using the inequality

$$
\|g\|_{2}^{2} \leq \frac{2}{N}\|\nabla g\|_{2}\|x g\|_{2} \quad \text { if } g \in H^{1}\left(\mathbb{R}^{N}\right), x g \in L^{2}\left(\mathbb{R}^{N}\right)
$$

and noticing that $\|u(\cdot, t)\|_{2}=\left\|u_{0}\right\|_{2}$, we have

$$
\lim _{t \rightarrow T_{\max }}\|\nabla u\|_{2}=+\infty
$$

Consequently,

$$
\lim _{t \rightarrow T_{\max }}\|u\|_{\Sigma}=+\infty
$$

(2) Similar to (46), we can get

$$
0 \leq J(t) \leq J(0)+J^{\prime}(0) t
$$

which implies that the solution will blow up in a finite time $T_{\max } \leq J(0) /-J^{\prime}(0)$.

\section{The Sufficient Conditions on Global Existence and Blowup in Finite Time}

In this section, we will prove Theorems 3 and 4 , which give some sufficient conditions on global existence and blowup of the solution to (1).

We would like to give some examples of $V(x), f\left(x,|u|^{2}\right)$, and $W(x)$. It is easy to verify that they satisfy the conditions of Theorem 3.

Example 11. Consider that $V(x)=|x|^{2}, W(x)=e^{-\pi|x|^{2}}$, and $f\left(x,|u|^{2}\right)=b|u|^{2 p}$ with $b$ a real constant and $0<p<2 / N$.

Example 12. Consider that $V(x)=|x|^{2}, W(x)=|x|^{2} /(1+$ $\left.|x|^{2}\right)$, and $f\left(x,|u|^{2}\right)=b|u|^{2 p} \ln \left(1+|u|^{2}\right)$ with $b$ a real constant and $0<p<2 / N$.
Proof of Theorem 3. Letting $W^{+}(x)=W_{1}(x)+W_{2}(x)$, where $W_{1} \in L^{\infty}\left(\mathbb{R}^{N}\right)$ and $W_{2} \in L^{q}\left(\mathbb{R}^{N}\right)$ with $q>N / 2$, using Hölder's and Young's inequalities, we obtain

$$
\int_{\mathbb{R}^{N}}\left(W_{2}(x) \star(u v)\right) w z d x \leq\left\|W_{2}\right\|_{L^{q}}\|u\|_{L^{r}}\|v\|_{L^{r}}\|w\|_{L^{r}}\|z\|_{L^{r}}
$$

with $r=4 q /(2 q-1)$. Specifically, we have

$$
\int_{\mathbb{R}^{N}}\left(W_{2}(x) \star|u|^{2}\right)|u|^{2} d x \leq\left\|W_{2}\right\|_{L^{q}}\|u\|_{L^{r}}^{4} .
$$

Using (53) and Gagliardo-Nirenberg's inequality, we get

$$
\begin{aligned}
\frac{1}{4} \int_{\mathbb{R}^{N}} & \left(W(x) \star|u|^{2}\right)|u|^{2} d x \\
\leq & \left\|W_{1}\right\|_{L^{\infty}}\|u\|_{L^{2}}^{4}+\left\|W_{2}\right\|_{L^{q}}\|u\|_{L^{4 q /(2 q-1)}}^{4} \\
\leq & \left\|W_{1}\right\|_{L^{\infty}}\|u\|_{L^{2}}^{4} \\
& +C\left\|W_{2}\right\|_{L^{q}}\|\nabla u\|_{L^{2}}^{N / q}\|u\|_{L^{2}}^{(4 q-N) / q} .
\end{aligned}
$$

Using Young's inequality, from (54), we have

$$
\begin{aligned}
& C\left\|W_{2}\right\|_{L^{q}}\|\nabla u\|_{L^{2}}^{N / q}\|u\|_{L^{2}}^{(4 q-N) / q} \\
& \quad \leq \varepsilon\|\nabla u\|_{L^{2}}^{2}+C\left(\varepsilon,\left\|W_{2}\right\|_{L^{q}}\right)\|u\|_{L^{2}}^{(8 q-2 N) /(2 q-N)}
\end{aligned}
$$

for some $\varepsilon>0$. Noticing that $F\left(x,|u|^{2}\right) \leq c_{1}|u|^{2}+c_{2}|u|^{2 p+2}$, using Gagliardo-Nirenberg's inequality and (55) with $\varepsilon=1 / 4$, we get

$$
\begin{aligned}
E\left(u_{0}\right)= & \frac{1}{2}\left(\int_{\mathbb{R}^{N}}\left\{\left|\nabla u_{0}\right|^{2}+V(x)\left|u_{0}\right|^{2}-F\left(x,\left|u_{0}\right|^{2}\right)\right\} d x\right) \\
& -\frac{1}{4} \int_{\mathbb{R}^{N}}\left(W(x) \star\left|u_{0}\right|^{2}\right)\left|u_{0}\right|^{2} d x \\
= & \frac{1}{2}\left(\int_{\mathbb{R}^{N}}\left\{|\nabla u|^{2}+V(x)|u|^{2}-F\left(x,|u|^{2}\right)\right\} d x\right) \\
& -\frac{1}{4} \int_{\mathbb{R}^{N}}\left(W(x) \star|u|^{2}\right)|u|^{2} d x \\
\geq & \frac{1}{2}\left(\int_{\mathbb{R}^{N}}\left\{|\nabla u|^{2}+V(x)|u|^{2}-c_{1}|u|^{2}-c_{2}|u|^{2 p+2}\right\} d x\right) \\
& -\left\|W_{1}\right\|_{L^{\infty}}\|u\|_{L^{2}}^{4}-C\left\|W_{2}\right\|_{L^{q}}\|\nabla u\|_{L^{2}}^{N / q}\|u\|_{L^{2}}^{(4 q-N) / q} \\
\geq & \frac{1}{2}\left(\int_{\mathbb{R}^{N}}\left\{|\nabla u|^{2}+V(x)|u|^{2}-c_{1}|u|^{2}\right\} d x\right) \\
& -c_{2} C_{N}\left(\int_{\mathbb{R}^{N}}|\nabla u|^{2} d x\right)^{p N / 2}\left(\int_{\mathbb{R}^{N}}|u|^{2} d x\right)^{(2+p(2-N)) / 2} \\
& -\left\|W_{1}\right\|_{L^{\infty}}\|u\|_{L^{2}}^{4}-\frac{1}{4}\|\nabla u\|_{L^{2}}^{2}-C\|u\|_{L^{2}}^{(8 q-2 N) /(2 q-N)} .
\end{aligned}
$$


Since $\|u\|_{2}=\left\|u_{0}\right\|_{2}$, from (56), we can obtain

$$
\begin{aligned}
4 E\left(u_{0}\right) & +C\left\|u_{0}\right\|_{L^{2}}^{2}+C\left\|u_{0}\right\|_{L^{2}}^{4} \\
& +C\left\|u_{0}\right\|_{L^{2}}^{(8 q-2 N) /(2 q-N)} \\
\geq & \int_{\mathbb{R}^{N}} V(x)|u|^{2} d x+\int_{\mathbb{R}^{N}}|\nabla u|^{2} d x \\
& \times\left(1-c\left\{\int_{\mathbb{R}^{N}}|\nabla u|^{2} d x\right\}^{(p N / 2)-1}\right) .
\end{aligned}
$$

Since $p<2 / N$ means that $(p N / 2)-1<0$, (57) implies that $\|u\|_{\Sigma}^{2}$ is always controlled by $4 E\left(u_{0}\right)+C\left\|u_{0}\right\|_{L^{2}}^{2}+C\left\|u_{0}\right\|_{L^{2}}^{4}+$ $C\left\|u_{0}\right\|_{L^{2}}^{(8-2 N) /(2 q-N)}$. That is, the solution of (1) exists globally.

We would like to give some examples of $V(x), W(x)$, and $f\left(x,|u|^{2}\right)$. It is easy to verify that they satisfy the conditions of Theorem 4 .

Example 13. Consider that $V(x)=|x|^{2}, W(x)=|x|^{-2}$, and $f\left(x,|u|^{2}\right)=b|u|^{2 p}$ with $b>0$ and $p>2 / N$ with $N \geq 3$.

Example 14. Consider that $V(x)=|x|^{2}, W(x)=|x|^{-2}$, and $f\left(x,|u|^{2}\right)=b|u|^{2 p} \ln \left(1+|u|^{2}\right)$ with $b>0$ and $p \geq 2 / N$ with $N \geq 3$.

Proof of Theorem 4. Set

$$
y(t)=J^{\prime}(t)=4 \mathfrak{\Im} \int_{\mathbb{R}^{N}}(x \cdot \nabla u) \bar{u} d x .
$$

Using (18)-(20), we have

$$
\begin{aligned}
y^{\prime}(t)= & 8 \int_{\mathbb{R}^{N}}|\nabla u|^{2} d x-4 \int_{\mathbb{R}^{N}}(x \cdot \nabla V(x))|u|^{2} d x \\
& +4 N \int_{\mathbb{R}^{N}}\left[F\left(x,|u|^{2}\right)-|u|^{2} f\left(x,|u|^{2}\right)\right] d x \\
& +2 \int_{\mathbb{R}^{N}}\left\{(x \cdot \nabla W(x)) \star|u|^{2}\right\}|u|^{2} d x \\
= & 16 E(u)+4 \int_{\mathbb{R}^{N}}\left([-2 V(x)-(x \cdot \nabla V(x))]|u|^{2}\right. \\
& +2 \int_{\mathbb{R}^{N}}\left[\{2 W(x)+(x \cdot \nabla W(x))\} \star|u|^{2}\right]|u|^{2} d x \\
\leq & 16 E(u)=16 E\left(u_{0}\right)<0 .
\end{aligned}
$$

From (58) and (59), we obtain

$$
\begin{aligned}
\|x u(x, t)\|_{L^{2}}^{2} \leq & \left\|x u_{0}\right\|_{L^{2}}^{2} \\
& +4 t \Im \int_{\mathbb{R}^{N}} \bar{u}_{0}\left(x \cdot \nabla u_{0}\right) d x+8 t^{2} E\left(u_{0}\right) .
\end{aligned}
$$

Since $\|x u(x, t)\|_{L^{2}}^{2} \geq 0$, whether (1) or (2), (60) will be absurd for $t>0$ large enough. Therefore, the solution of (1) will blow up in finite time.

\section{The Sharp Threshold for Global Existence and Blowup of the Solution to (1) with $V(x) \equiv 0$ and $W \in L^{q}\left(\mathbb{R}^{N}\right)$ with $N / 4<q<N / 2$}

In this section, we will establish the sharp threshold for global existence and blowup of the solution to (1) with $V(x) \equiv 0$ and $W \in L^{q}\left(\mathbb{R}^{N}\right)$ with $N / 4<q<N / 2$.

Before giving the proof of Theorem 5, we would like to give some examples of $f\left(x,|u|^{2}\right)$ and $W(x)$. It is easy to verify that they satisfy the conditions of Theorem 5 .

Example 15. Consider that $W(x) \equiv 0, f\left(x,|u|^{2}\right)=c|u|^{2 q_{1}}+$ $d|u|^{2 q_{2}}$ with $c<0, d>0$ and $q_{2}>2 / N, q_{2}>q_{1}>0$.

Example 16. Consider that $W(x) \equiv 0, f\left(x,|u|^{2}\right)=$ $b|u|^{2 p} \ln \left(1+|u|^{2}\right)$ with $b>0$ and $p>2 / N$.

Example 17. Let $f\left(x,|u|^{2}\right)$ be one of those in Examples 15 and 16. Let

$$
W(x)= \begin{cases}\frac{1}{|x|^{N l}}, & |x| \leq 1 \\ \varphi(x), & 1 \leq|x| \leq 2, \\ \frac{1}{|x|^{K}}, & |x| \geq 2\end{cases}
$$

where $2<N l<N / q<K$ and $\varphi(x)$ satisfies

$$
N l \varphi(x)+(x \cdot \nabla \varphi) \leq 0 \leq c_{3} \varphi(x)+(x \cdot \nabla \varphi)
$$

when $1 \leq|x| \leq 2$ and makes $W(x)$ smooth. Obviously, $W \in$ $L^{q}\left(\mathbb{R}^{N}\right)$.

Proof of Theorem 5. We will proceed in four steps.

Step 1. We will prove $d_{I}>0 . u \in H^{1}\left(\mathbb{R}^{N}\right) \backslash\{0\}$ and $Q(u)=0$ mean that

$$
\begin{aligned}
2 \int_{\mathbb{R}^{N}}|\nabla u|^{2} d x= & N \int_{\mathbb{R}^{N}}\left[|u|^{2} f\left(x,|u|^{2}\right)-F\left(x,|u|^{2}\right)\right] d x \\
& -\frac{1}{2} \int_{\mathbb{R}^{N}}\left\{(x \cdot \nabla W(x)) \star|u|^{2}\right\}|u|^{2} d x \\
\leq & \frac{N(l+1)}{l} \int_{\mathbb{R}^{N}}\left[c_{1}|u|^{2 p_{1}+2}+c_{2}|u|^{2 p_{2}+2}\right] d x \\
& +C \int_{\mathbb{R}^{N}}\left(W(x) \star|u|^{2}\right)|u|^{2} d x \\
\leq & C\|u\|_{2 p_{1}+2}^{2 p_{1}+2}+C\|u\|_{2 p_{2}+2}^{2 p_{2}+2} \\
& +C\|W\|_{L^{q}}\|u\|_{L^{4 q /(2 q-1)} .}^{4}
\end{aligned}
$$


Using Gagliardo-Nirenberg's and Hölder's inequalities, we can get

$$
\begin{aligned}
2 \leq & C\left(\|\nabla u\|_{2}^{2}\right)^{N p_{1} / 2}\left(\|u\|_{2}^{2}\right)^{p_{1}+1-\left(N p_{1} / 2\right)} \\
+ & C\left(\|\nabla u\|_{2}^{2}\right)^{N p_{2} / 2}\left(\|u\|_{2}^{2}\right)^{p_{2}+1-\left(N p_{2}\right) / 2} \\
& +C\left(\|\nabla u\|_{2}^{2}\right)^{N / 2 q}\left(\|u\|_{2}^{2}\right)^{(4 q-N) / 2 q} \\
\leq & C\left\{\left(\|\nabla u\|_{2}^{2}+\|u\|_{2}^{2}\right)^{p_{1}+1}+\left(\|\nabla u\|_{2}^{2}+\|u\|_{2}^{2}\right)^{p_{2}+1}\right. \\
& \left.+\left(\|\nabla u\|_{2}^{2}+\|u\|_{2}^{2}\right)^{2}\right\} .
\end{aligned}
$$

That is,

$$
\|\nabla u\|_{2}^{2}+\|u\|_{2}^{2} \geq C>0
$$

if $Q(u)=0$ and $u \in H^{1}\left(\mathbb{R}^{N}\right) \backslash\{0\}$.

On the other hand, if $Q(u)=0$, we have

$$
\begin{aligned}
2 \int_{\mathbb{R}^{N}}|\nabla u|^{2} d x= & N \int_{\mathbb{R}^{N}}\left[|u|^{2} f\left(x,|u|^{2}\right)-F\left(x,|u|^{2}\right)\right] d x \\
& -\frac{1}{2} \int_{\mathbb{R}^{N}}\left\{(x \cdot \nabla W(x)) \star|u|^{2}\right\}|u|^{2} d x \\
\geq & N l \int_{\mathbb{R}^{N}} F\left(x,|u|^{2}\right) d x \\
& +\frac{N l}{2} \int_{\mathbb{R}^{N}}\left\{W(x) \star|u|^{2}\right\}|u|^{2} d x
\end{aligned}
$$

that is,

$$
\begin{aligned}
& -\frac{1}{2} \int_{\mathbb{R}^{N}} F\left(x,|u|^{2}\right) d x \\
& \quad-\frac{1}{4} \int_{\mathbb{R}^{N}}\left\{W(x) \star|u|^{2}\right\}|u|^{2} d x \geq-\frac{1}{N l} \int_{\mathbb{R}^{N}}|\nabla u|^{2} d x .
\end{aligned}
$$

Using (67), we can obtain

$$
\begin{aligned}
\omega\|u\|_{2}^{2}+E(u)= & \omega\|u\|_{2}^{2}+\frac{1}{2} \int_{\mathbb{R}^{N}}|\nabla u|^{2} d x \\
& -\frac{1}{2} \int_{\mathbb{R}^{N}} F\left(x,|u|^{2}\right) d x \\
& -\frac{1}{4} \int_{\mathbb{R}^{N}}\left\{W(x) \star|u|^{2}\right\}|u|^{2} d x \\
\geq & \omega\|u\|_{2}^{2}+\left(\frac{1}{2}-\frac{1}{N l}\right) \int_{\mathbb{R}^{N}}|\nabla u|^{2} d x \\
\geq & \min \left\{\omega,\left(\frac{1}{2}-\frac{1}{N l}\right)\right\} \times\left(\|\nabla u\|_{2}^{2}+\|u\|_{2}^{2}\right) \\
\geq & C>0
\end{aligned}
$$

from (65). Hence

$$
d_{I}>0
$$

Step 2. Denote

$$
\begin{aligned}
& K_{+}=\left\{u \in H^{1}\left(\mathbb{R}^{N}\right) \backslash\{0\}, Q(u)>0, \omega\|u\|_{2}^{2}+E(u)<d_{I}\right\}, \\
& K_{-}=\left\{u \in H^{1}\left(\mathbb{R}^{N}\right) \backslash\{0\}, Q(u)<0, \omega\|u\|_{2}^{2}+E(u)<d_{I}\right\} .
\end{aligned}
$$

We will prove that $K_{+}$and $K_{-}$are invariant sets of (1) with $V(x) \equiv 0$ and $W \in L^{q}\left(\mathbb{R}^{N}\right)$ with $N / 4<q<N / 2$. That is, we need to show that $u(\cdot, t) \in \mathscr{K}$ for all $t \in\left(0, T_{\text {max }}\right)$ if $u_{0} \in K_{+}$. Since $\|u\|_{2}$ and $E(u)$ are conservation quantities for (1), we have

$$
u(\cdot, t) \in H^{1}\left(\mathbb{R}^{N}\right) \backslash\{0\}, \omega\|u(\cdot, t)\|_{2}^{2}+E(u(\cdot, t))<d_{I}
$$

for all $t \in\left(0, T_{\max }\right)$ if $u_{0} \in K_{+}$. We need to prove that $Q(u(\cdot, t))>0$. Otherwise, assume that there exists a $t_{1} \in$ $\left(0, T_{\max }\right)$ satisfying $Q\left(u\left(\cdot, t_{1}\right)\right)=0$ by the continuity. Note that (71) implies

$$
\omega\|u(\cdot, t)\|_{2}^{2}+E\left(u\left(\cdot, t_{1}\right)\right)<d_{I} .
$$

However, the inequality above and $Q\left(u\left(\cdot, t_{1}\right)\right)=0$ are contradictions to the definition of $d_{I}$. Therefore, $Q(u(\cdot, t))>$ 0 . Consequently, $(71)$ and $Q(u(\cdot, t))>0$ imply that $u(\cdot, t) \in$ $K_{+}$. That is, $K_{+}$is an invariant set of (1) with $V(x) \equiv 0$ and $W \in L^{q}\left(\mathbb{R}^{N}\right)$ with $N / 4<q<N / 2$. Similarly, we can prove that $K_{-}$is also an invariant set of (1) with $V(x) \equiv 0$ and $W \in L^{q}\left(\mathbb{R}^{N}\right)$ with $N / 4<q<N / 2$.

Step 3. Assume that $Q\left(u_{0}\right)>0$ and $\omega\left\|u_{0}\right\|_{2}^{2}+E\left(u_{0}\right)<d_{I}$. By the results of Step 2 , we have $Q(u(\cdot, t))>0$ and $\omega\|u(\cdot, t)\|_{2}^{2}+$ $E(u(\cdot, t))<d_{I}$. That is,

$$
\begin{aligned}
&-2\|\nabla u(\cdot, t)\|_{2}^{2}<-N \int_{\mathbb{R}^{N}}\left[|u|^{2} f\left(x,|u|^{2}\right)-F\left(x,|u|^{2}\right)\right] d x \\
&+\frac{1}{2} \int_{\mathbb{R}^{N}}\left\{(x \cdot \nabla W(x)) \star|u|^{2}\right\}|u|^{2} d x \\
&<-N l \int_{\mathbb{R}^{N}} F\left(x,|u|^{2}\right) d x \\
&-\frac{N l}{2} \int_{\mathbb{R}^{N}}\left\{W(x) \star|u|^{2}\right\}|u|^{2} d x \\
& d_{I}>\omega\|u(\cdot, t)\|_{2}^{2}+\frac{1}{2}\|\nabla u(\cdot, t)\|_{2}^{2} \\
&-\frac{1}{2} \int_{\mathbb{R}^{N}} F\left(x,|u|^{2}\right) d x \\
&-\frac{1}{4} \int_{\mathbb{R}^{N}}\left\{W(x) \star|u|^{2}\right\}|u|^{2} d x .
\end{aligned}
$$

The two inequalities imply that

$$
\omega\|u(\cdot, t)\|_{2}^{2}+\left(\frac{1}{2}-\frac{1}{N l}\right)\|\nabla u(\cdot, t)\|_{2}^{2}<d_{I},
$$

which means that

$$
\|u(\cdot, t)\|_{H^{1}\left(\mathbb{R}^{N}\right)}<\infty ;
$$

that is, the solution exists globally. 
Step 4. Assume that $Q\left(u_{0}\right)<0$ and $\omega\left\|u_{0}\right\|_{2}^{2}+E\left(u_{0}\right)<d_{I}$. By the results of Step 2, we obtain $Q(u(\cdot, t))<0$ and $\omega\|u(\cdot, t)\|_{2}^{2}+$ $E(u(\cdot, t))<d_{I}$. Hence we get

$$
J^{\prime \prime}(t)=4 Q(u)<0, J^{\prime}(0)=4 \mathfrak{I} \int_{\mathbb{R}^{N}}\left(x \cdot \nabla u_{0}\right) \bar{u}_{0} d x<0 .
$$

By the results of Proposition 10, the solution will blow up in finite time.

As a corollary of Theorem 5, we obtain the sharp threshold for global existence and blowup of the solution of (8) as follows.

Corollary 18. Assume that $f(x, 0)=0$ and (23). Let $\omega$ be a positive constant satisfying

$$
d_{I}^{\prime}:=\inf _{\left\{u \in \Sigma \mid\{0\} ; Q_{1}(u)=0\right\}}\left(\omega\|u\|_{2}^{2}+E(u)\right)>0 .
$$

Here

$$
\begin{aligned}
Q_{1}(u):= & 2 \int_{\mathbb{R}^{N}}|\nabla u|^{2} d x \\
& +N \int_{\mathbb{R}^{N}}\left[F\left(x,|u|^{2}\right)-|u|^{2} f\left(x,|u|^{2}\right)\right] d x .
\end{aligned}
$$

Suppose that $u_{0} \in H^{1}\left(\mathbb{R}^{N}\right)$ satisfies

$$
\omega\left\|u_{0}\right\|_{2}^{2}+E\left(u_{0}\right)<d_{I}^{\prime} .
$$

Then

(1) if $Q_{1}\left(u_{0}\right)>0$, the solution of (8) exists globally;

(2) if $Q_{1}\left(u_{0}\right)<0,|x| u_{0} \in L^{2}\left(\mathbb{R}^{N}\right)$, and $\mathfrak{\Im} \int_{\mathbb{R}^{N}}(x$. $\left.\nabla u_{0}\right) \bar{u}_{0} d x<0$, the solution of (8) blows up in finite time.

Remark 19. In Theorem 1.5 of [10], Tao et al. proved the following.

Assume that $u(x, t)$ is a solution of (8) with $f\left(x,|u|^{2}\right) u=$ $\mu|u|^{p_{1}} u+v|u|^{p_{2}} u$, where $\mu>0, v>0,4 / N \leq p_{1}<p_{2} \leq 4 /(N-$ 2) with $N \geq 3, \mathfrak{J} \int_{\mathbb{R}^{N}}\left(x \cdot \nabla u_{0}\right) \bar{u}_{0} d x<0,|x| u_{0} \in L^{2}\left(\mathbb{R}^{N}\right)$, and $E\left(u_{0}\right)<0$. Then blowup occurs.

Corollary 18 covers the result above under some conditions. In fact, if $f\left(x,|u|^{2}\right) u=\mu|u|^{p_{1}} u+v|u|^{p_{2}} u$, then

$$
\begin{aligned}
Q_{1}(u)= & 4 E(u)-\frac{\left(N p_{1}-4\right) \mu}{\left(p_{1}+2\right)}\|u\|_{p_{1}+2}^{p_{1}+2} \\
& -\frac{\left(N p_{2}-4\right) v}{\left(p_{2}+2\right)}\|u\|_{p_{2}+2}^{p_{2}+2} \leq E(u) ;
\end{aligned}
$$

hence $E\left(u_{0}\right)<0$ implies that $Q_{1}\left(u_{0}\right)<0$. That is, our blowup condition is weaker than theirs. On the other hand, our conclusion is still true if $0<E\left(u_{0}\right)<d_{I}^{\prime}-\omega\left\|u_{0}\right\|_{2}^{2}$ with $Q_{1}\left(u_{0}\right)<0, \mathfrak{J} \int_{\mathbb{R}^{N}}\left(x \cdot \nabla u_{0}\right) \bar{u}_{0} d x<0$, and $|x| u_{0} \in$ $L^{2}\left(\mathbb{R}^{N}\right)$. In other words, our result is stronger than theirs if $\omega\left\|u_{0}\right\|_{2}^{2}+E\left(u_{0}\right)<d_{I}^{\prime}$ with $Q_{1}\left(u_{0}\right)<0, \mathfrak{\Im} \int_{\mathbb{R}^{N}}\left(x \cdot \nabla u_{0}\right) \bar{u}_{0} d x<0$, and $|x| u_{0} \in L^{2}\left(\mathbb{R}^{N}\right)$.

\section{Sharp Threshold for the Blowup and Global Existence of the Solution to (1)}

Theorem 7 is inspired by [5], but it extends the results to more general case. We need subtle estimates and more sophisticated analysis in the proof.

First, we would like to give some examples of $V(x)$, $f\left(x,|u|^{2}\right)$, and $W(x)$. It is easy to verify that they satisfy the conditions of Theorem 7 .

Example 20. Consider that $V(x)=|x|^{2}, W(x)=a|x|^{-K}$ with $2<N l<K<N / q<4$ for $x \in \mathbb{R}^{N}$ and $f\left(x,|u|^{2}\right)=b|u|^{2 p_{1}}+$ $c|u|^{2 p_{2}}$ with $a \geq 0, b>0, c>0$, and $p_{2}>p_{1}>2 / N$.

Example 21. Consider that $V(x)=|x|^{2}, W(x)=a|x|^{-K}$ with $2<N l<K<N / q<4$ for $x \in \mathbb{R}^{N}$ and $f\left(x,|u|^{2}\right)=c|u|^{2 q_{1}}+$ $d|u|^{2 q_{2}}$ with $a \geq 0, c$ is a real number, $d>0$, and $q_{2}>2 / N$, $q_{2}>q_{1}>0$.

Example 22. Consider that $V(x)=|x|^{2} /\left(1+|x|^{2}\right), W(x)=$ $a|x|^{-K}$ with $2<N l<K<N / q<4$ for $x \in \mathbb{R}^{N}$ and $f\left(x,|u|^{2}\right)=b|u|^{2 p} \ln \left(1+|u|^{2}\right)$ with $a \geq 0, b>0$, and $p>2 / N$.

5.1. Some Invariant Manifolds. In this subsection, we will prove that $d_{\mathcal{N}}, d_{\mathscr{M}}, d_{I I}>0$ and construct some invariant manifolds.

Proposition 23. Assume that the conditions of Theorem 7 hold. Then $d_{N}>0$.

Proof. Assume that $u \in \Sigma \backslash\{0\}$ satisfying $S_{\omega}(u)=0$. Using Gagliardo-Nirenberg's and Young's inequalities, we have

$$
\begin{aligned}
2 \omega\|u\|_{2}^{2} & +\int_{\mathbb{R}^{N}}\left[|\nabla u|^{2}+V(x)|u|^{2}\right] d x \\
= & \int_{\mathbb{R}^{N}}|u|^{2} f\left(x,|u|^{2}\right) d x \\
& +\int_{\mathbb{R}^{N}}\left(W(x) \star|u|^{2}\right)|u|^{2} d x \\
\leq & \frac{l+1}{l} \int_{\mathbb{R}^{N}}\left[c_{1}|u|^{2 p_{1}+2}+c_{2}|u|^{2 p_{2}+2}\right] d x \\
& +\left\|W_{1}\right\|_{L^{\infty}}\|u\|_{2}^{4}+\left\|W_{2}\right\|_{L^{q}}\|u\|_{L^{4 q /(2 q-1)}}^{4} \\
\leq & C\left(\|\nabla u\|_{2}^{2}\right)^{N p_{1} / 2}\left(\|u\|_{2}^{2}\right)^{p_{1}+1-\left(N p_{1} / 2\right)} \\
& +C\left(\|\nabla u\|_{2}^{2}\right)^{N p_{2} / 2}\left(\|u\|_{2}^{2}\right)^{p_{2}+1-\left(N p_{2} / 2\right)} \\
& +\left\|W_{1}\right\|_{L^{\infty}}\|u\|_{2}^{4} \\
& +C\left\|W_{2}\right\|_{L^{q}}\|\nabla u\|_{2}^{N / q}\|u\|_{2}^{(4 q-N) / q} \\
\leq & C\left(\|\nabla u\|_{2}^{2}\right)^{N p_{1} / 2}\left(\|u\|_{2}^{2}\right)^{p_{1}+1-\left(N p_{1} / 2\right)}
\end{aligned}
$$




$$
\begin{aligned}
& +C\left(\|\nabla u\|_{2}^{2}\right)^{N p_{2} / 2}\left(\|u\|_{2}^{2}\right)^{p_{2}+1-\left(N p_{2} / 2\right)} \\
& +C\|u\|_{2}^{4}+\|\nabla u\|_{2}^{4}+C\left(\left\|W_{2}\right\|_{L^{q}}\right)\|u\|_{2}^{4} .
\end{aligned}
$$

Using Hölder's inequality, from (81), we can obtain

$$
\begin{aligned}
2 \omega\|u\|_{2}^{2} & +\int_{\mathbb{R}^{N}}\left[|\nabla u|^{2}+V(x)|u|^{2}\right] d x \\
\leq & C\left(2 \omega\|u\|_{2}^{2}+\int_{\mathbb{R}^{N}}\left[|\nabla u|^{2}+V(x)|u|^{2}\right] d x\right)^{p_{1}+1} \\
& +C\left(2 \omega\|u\|_{2}^{2}+\int_{\mathbb{R}^{N}}\left[|\nabla u|^{2}+V(x)|u|^{2}\right] d x\right)^{p_{2}+1} \\
& +C\left(2 \omega\|u\|_{2}^{2}+\int_{\mathbb{R}^{N}}\left[|\nabla u|^{2}+V(x)|u|^{2}\right] d x\right)^{2} .
\end{aligned}
$$

Equation (82) implies that

$$
2 \omega\|u\|_{2}^{2}+\int_{\mathbb{R}^{N}}\left[|\nabla u|^{2}+V(x)|u|^{2}\right] d x \geq C>0
$$

for some positive constant $C$.

On the other hand, if $S_{\omega}(u)=0$, we get

$$
\begin{aligned}
\omega\|u\|_{2}^{2}+ & \frac{1}{2} \int_{\mathbb{R}^{N}}\left(|\nabla u|^{2}+V(x)|u|^{2}\right) d x \\
= & \frac{1}{2} \int_{\mathbb{R}^{N}} f\left(x,|u|^{2}\right)|u|^{2} d x \\
& +\frac{1}{2} \int_{\mathbb{R}^{N}}\left(W(x) \star|u|^{2}\right)|u|^{2} d x \\
\geq & \min (l+1,2)\left(\frac{1}{2} \int_{\mathbb{R}^{N}} F\left(x,|u|^{2}\right) d x\right. \\
& \left.+\frac{1}{4} \int_{\mathbb{R}^{N}}\left(W(x) \star|u|^{2}\right)|u|^{2} d x\right) .
\end{aligned}
$$

From (84), we obtain

$$
\begin{aligned}
I_{\omega}(u)= & \omega\|u\|_{2}^{2} \\
& +\frac{1}{2} \int_{\mathbb{R}^{N}}\left[|\nabla u|^{2}+V(x)|u|^{2}-F\left(x,|u|^{2}\right)\right] d x \\
& -G\left(|u|^{2}\right) \\
\geq & \min \left(\frac{l}{2(l+1)}, \frac{1}{4}\right) \\
& \times\left(2 \omega\|u\|_{2}^{2}+\int_{\mathbb{R}^{N}}\left[|\nabla u|^{2}+V(x)|u|^{2}\right] d x\right) \\
\geq & C>0 .
\end{aligned}
$$

Consequently,

$$
d_{\mathcal{N}}=\inf _{\mathcal{N}} I_{\omega}(u)>C>0 .
$$

Now, we will give some properties of $I_{\omega}(u), S_{\omega}(u)$, and $Q(u)$. We have a proposition as follows.

Proposition 24. Assume that $Q(u)$ and $S_{\omega}(u)$ are defined by (22) and (28). Then one has the following.

(i) There at least exists a $w^{\star} \in \Sigma \backslash\{0\}$ such that

$$
S_{\omega}\left(w^{\star}\right)=0, \quad Q\left(w^{\star}\right)=0 .
$$

(ii) There at least exists a $u^{*} \in \Sigma \backslash\{0\}$ such that

$$
S_{\omega}\left(u^{*}\right)<0, \quad Q\left(u^{*}\right)=0 .
$$

Proof. (i) Noticing the assumptions on $V(x), W(x)$, and $f\left(x,|u|^{2}\right)$, similar to the proof of Theorem 1.7 in [17], it is easy to prove that there exists a $w^{\star} \in \Sigma \backslash\{0\}$ satisfying

$$
\begin{aligned}
2 \omega w^{\star}+ & V(x) w^{\star}-\Delta w^{\star}=f\left(x,\left|w^{\star}\right|^{2}\right) w^{\star} \\
& +\left(W(x) \star\left|w^{\star}\right|^{2}\right) w^{\star} \quad \text { in } \mathbb{R}^{N} .
\end{aligned}
$$

Multiplying (89) by $w^{\star}$ and integrating over $\mathbb{R}^{N}$ by part, we can get $S_{\omega}\left(w^{\star}\right)=0$.

Multiplying (89) by $\left(x \cdot \nabla w^{\star}\right)$ and integrating over $\mathbb{R}^{N}$ by part, we obtain Pohozaev's identity:

$$
\begin{aligned}
N \omega\left\|w^{\star}\right\|_{2}^{2} & +\frac{N-2}{2} \int_{\mathbb{R}^{N}}\left|\nabla w^{\star}\right|^{2} d x \\
& +\frac{N}{2} \int_{\mathbb{R}^{N}} V(x)\left|w^{\star}\right|^{2} d x \\
& +\frac{1}{2} \int_{\mathbb{R}^{N}}(x \cdot \nabla V(x))\left|w^{\star}\right|^{2} d x \\
= & \frac{N}{2} \int_{\mathbb{R}^{N}} F\left(x,\left|w^{\star}\right|^{2}\right) d x \\
& +\frac{N}{2} \int_{\mathbb{R}^{N}}\left(W(x) \star\left|w^{\star}\right|^{2}\right)\left|w^{\star}\right|^{2} d x \\
& +\frac{1}{2} \int_{\mathbb{R}^{N}}\left\{(x \cdot \nabla W(x)) \star\left|w^{\star}\right|^{2}\right\}\left|w^{\star}\right|^{2} d x .
\end{aligned}
$$

From $S_{\omega}\left(w^{\star}\right)=0$ and $(90)$, we can get $Q\left(w^{\star}\right)=0$. 
(ii) Letting $v_{k, \lambda}(x)=k w^{\star}(\lambda x)$ for $k>0$ and $\lambda>0$, we can obtain

$$
\begin{aligned}
S_{\omega}\left(v_{k, \lambda}\right)= & 2 \omega k^{2} \int_{\mathbb{R}^{N}}\left|w^{\star}(\lambda x)\right|^{2} d x \\
& +k^{2} \int_{\mathbb{R}^{N}}\left|\nabla w^{\star}(\lambda x)\right|^{2} d x \\
& +k^{2} \int_{\mathbb{R}^{N}} V(x)\left|w^{\star}(\lambda x)\right|^{2} d x \\
& -k^{2} \int_{\mathbb{R}^{N}}\left|w^{\star}(\lambda x)\right|^{2} f\left(x, k^{2}\left|w^{\star}(\lambda x)\right|^{2}\right) d x \\
& -k^{4} \int_{\mathbb{R}^{N}}\left(W(x) \star\left|w^{\star}(\lambda x)\right|^{2}\right)\left|w^{\star}(\lambda x)\right|^{2} d x,
\end{aligned}
$$

$$
\begin{aligned}
Q\left(v_{k, \lambda}\right)= & 2 k^{2} \int_{\mathbb{R}^{N}}\left|\nabla w^{\star}(\lambda x)\right|^{2} d x \\
& -k^{2} \int_{\mathbb{R}^{N}}(x \cdot \nabla V(x))\left|w^{\star}(\lambda x)\right|^{2} d x \\
& -N \int_{\mathbb{R}^{N}}\left[k^{2}\left|w^{\star}(\lambda x)\right|^{2} f\left(x, k^{2}\left|w^{\star}(\lambda x)\right|^{2}\right)\right] \\
& -F\left(x, k^{2}\left|w^{\star}(\lambda x)\right|^{2}\right) d x \\
& +\frac{k^{4}}{2} \int_{\mathbb{R}^{N}}\left((x \cdot \nabla W(x)) \star\left|w^{\star}(\lambda x)\right|^{2}\right) \\
& \times\left|w^{\star}(\lambda x)\right|^{2} d x .
\end{aligned}
$$

Looking at $S_{\omega}\left(v_{k, \lambda}\right)$ and $Q\left(v_{k, \lambda}\right)$ as the functions of $(k, \lambda)$, setting $g(k, \lambda)=S_{\omega}\left(v_{k, \lambda}\right)$ and $\eta(k, \lambda)=Q\left(v_{k, \lambda}\right)$, we get that $g(1,1)=0$ and $\eta(1,1)=0$. We want to prove that there exists a pair of $(k, \lambda)$ such that $g(k, \lambda)=S_{\omega}\left(v_{k, \lambda}\right)<0$ and $\eta(k, \lambda)=Q\left(v_{k, \lambda}\right)=0$. Since $\eta(1,1)=0$, we know that the image of $\eta(k, \lambda)$ and the plane $\eta=0$ intersect in the space of $(k, \lambda, \eta)$ and form a curve $\eta(k, \lambda)=0$. Hence there exist many positive real number pairs $(k, \lambda)$ relying on $w^{\star}$ such that $Q\left(v_{k, \lambda}\right)=0$ near $(1,1)$ with $k>1$. On the other hand, under the assumptions of $V(x)$ and $W(x)$, it is easy to see that $g(k, 1)<0$ for any $k>1$. By the continuity, we can choose that a pair of $(k, \lambda)$ near $(1,1)$ with $k>1$ satisfies both $Q\left(v_{k, \lambda}\right)=0$ and $S_{\omega}\left(v_{k, \lambda}\right)<0$. Letting $u^{*}=v_{k, \lambda}$ for this $(k, \lambda)$, we get that $S_{\omega}\left(u^{*}\right)<0$ and $Q\left(u^{*}\right)=0$.

Proposition 24 means that $\mathscr{C} \mathscr{M}$ is not empty and $d_{\mathscr{M}}$ is well defined. Moreover, we have the following.

Proposition 25. Assume that the conditions of Theorem 7 hold. Then $d_{\mathscr{M}}>0$.
Proof. $u \in \Sigma \backslash\{0\}$ and $S_{\omega}(u)<0$ imply that

$$
\begin{aligned}
2 \omega \int_{\mathbb{R}^{N}}|u|^{2} d x+\int_{\mathbb{R}^{N}}\left[|\nabla u|^{2}+V(x)|u|^{2}\right] d x \\
<\int_{\mathbb{R}^{N}}|u|^{2} f\left(x,|u|^{2}\right) d x \\
\quad+\int_{\mathbb{R}^{N}}\left(W(x) \star|u|^{2}\right)|u|^{2} d x \\
\leq \frac{l+1}{l} \int_{\mathbb{R}^{N}}\left[c_{1}|u|^{2 p_{1}+2}+c_{2}|u|^{2 p_{2}+2}\right] d x \\
\quad+\left\|W_{1}\right\|_{L^{\infty}}\|u\|_{L^{2}}^{4} \\
\quad+C\left\|W_{1}\right\|_{L^{q}}\|\nabla u\|_{L^{2}}^{N / q}\|u\|_{L^{2}}^{(4 q-N) / q} .
\end{aligned}
$$

Similar to (81) and (82), from (93), we have

$$
2 \omega \int_{\mathbb{R}^{N}}|u|^{2} d x+\int_{\mathbb{R}^{N}}\left[|\nabla u|^{2}+V(x)|u|^{2}\right] d x \geq C>0 .
$$

On the other hand, if $Q(u)=0$, we have

$$
\begin{aligned}
2 \int_{\mathbb{R}^{N}}|\nabla u|^{2} d x-\int_{\mathbb{R}^{N}}(x \cdot \nabla V(x))|u|^{2} d x \\
=N \int_{\mathbb{R}^{N}}\left[|u|^{2} f\left(x,|u|^{2}\right)-F\left(x,|u|^{2}\right)\right] d x \\
\quad-\frac{1}{2} \int_{\mathbb{R}^{N}}\left\{(x \cdot \nabla W(x)) \star|u|^{2}\right\}|u|^{2} d x \\
\geq N l \int_{\mathbb{R}^{N}} F\left(x,|u|^{2}\right) d x \\
\quad-\frac{1}{2} \int_{\mathbb{R}^{N}}\left\{(x \cdot \nabla W(x)) \star|u|^{2}\right\}|u|^{2} d x ;
\end{aligned}
$$

that is,

$$
\begin{aligned}
-\frac{1}{2} \int_{\mathbb{R}^{N}} F\left(x,|u|^{2}\right) d x & \\
& +\frac{1}{4 N l} \int_{\mathbb{R}^{N}}\left\{(x \cdot \nabla W(x)) \star|u|^{2}\right\}|u|^{2} d x \\
\geq & -\frac{1}{N l} \int_{\mathbb{R}^{N}}|\nabla u|^{2} d x+\frac{1}{2 N l} \int_{\mathbb{R}^{N}}(x \cdot \nabla V(x))|u|^{2} d x
\end{aligned}
$$


Using (23), (35), (36), (94), and (96), we can get

$$
\begin{aligned}
I_{\omega}(u)= & \omega \int_{\mathbb{R}^{N}}|u|^{2} d x \\
& +\frac{1}{2} \int_{\mathbb{R}^{N}}\left[|\nabla u|^{2}+V(x)|u|^{2}-F\left(x,|u|^{2}\right)\right] d x \\
& -\frac{1}{4} \int_{\mathbb{R}^{N}}\left(W(x) \star|u|^{2}\right)|u|^{2} d x \\
\geq & \omega \int_{\mathbb{R}^{N}}|u|^{2} d x+\frac{N l-2}{2 N l} \int_{\mathbb{R}^{N}}|\nabla u|^{2} d x \\
& +\frac{1}{2 N l} \int_{\mathbb{R}^{N}}[N l V(x)+(x \cdot \nabla V(x))]|u|^{2} d x \\
& -\frac{1}{4 N l} \int_{\mathbb{R}^{N}}\left\{[N l W(x)+(x \cdot \nabla W(x))] \star|u|^{2}\right\} \\
\geq & C\left(2 \omega \int_{\mathbb{R}^{N}}|u|^{2} d x\right. \\
\geq & C>0 . \\
& \left.\quad\left[|\nabla u|_{\mathbb{R}^{N}}+V(x)|u|^{2}\right] d x\right) \\
& \left(x|u|^{2} d x\right.
\end{aligned}
$$

Consequently,

$$
d_{\mathscr{M}}=\inf _{\mathscr{C} \mathscr{M}} I_{\omega}(u)>C>0
$$

By the conclusions of Proposition 23 and Proposition 25, we have

$$
d_{I I}=\min \left\{d_{\mathscr{M}}, d_{\mathscr{N}}\right\}>0 .
$$

Now we define the following manifolds:

$$
\begin{gathered}
\mathscr{K}:=\left\{u \in \Sigma \backslash\{0\}: I_{\omega}(u)<d_{I I}, S_{\omega}(u)<0, Q(u)<0\right\} \\
\mathscr{K}_{+}:=\left\{u \in \Sigma \backslash\{0\}: I_{\omega}(u)<d_{I I}, S_{\omega}(u)<0, Q(u)>0\right\} \\
\mathscr{R}_{+}:=\left\{u \in \Sigma \backslash\{0\}: I_{\omega}(u)<d_{I I}, S_{\omega}(u)>0\right\}
\end{gathered}
$$

The following proposition will show some properties of $\mathscr{K}$, $\mathscr{K}_{+}$, and $\mathscr{R}_{+}$.

Proposition 26. Assume that the conditions of Theorem 7 hold. Then

(i) $\mathscr{K}, \mathscr{K}_{+}$, and $\mathscr{R}_{+}$are not empty;

(ii) $\mathscr{K}, \mathscr{K}_{+}$, and $\mathscr{R}_{+}$are invariant manifolds of (1).

Proof. (i) In order to prove $\mathscr{K}$ is not empty, we only need to find that there at least exists a $w \in \mathscr{K}$. For $w^{\star} \in \Sigma \backslash\{0\}$ satisfying $S_{\omega}\left(w^{\star}\right)=0$ and $Q\left(w^{\star}\right)=0$, letting $w_{\rho}=\rho w^{\star}$ for $\rho>0$, we have

$$
\begin{aligned}
& S_{\omega}\left(w_{\rho}\right)=\rho^{2} \int_{\mathbb{R}^{N}}\left\{2 \omega\left|w^{\star}\right|^{2}+\left|\nabla w^{\star}\right|^{2}+V(x)\left|w^{\star}\right|^{2}\right\} d x \\
& -\int_{\mathbb{R}^{N}} \rho^{2}\left|w^{\star}\right|^{2} f\left(x, \rho^{2}\left|w^{\star}\right|^{2}\right) d x \\
& -\rho^{4} \int_{\mathbb{R}^{N}}\left(W(x) \star\left|w^{\star}\right|^{2}\right)\left|w^{\star}\right|^{2} d x, \\
& Q\left(w_{\rho}\right)=\rho^{2} \int_{\mathbb{R}^{N}}\left(2\left|\nabla w^{\star}\right|^{2}-(x \cdot \nabla V(x))\left|w^{\star}\right|^{2}\right) d x \\
& +N \int_{\mathbb{R}^{N}}\left[F\left(x, \rho^{2}\left|w^{\star}\right|^{2}\right)\right. \\
& \left.-\rho^{2}\left|w^{\star}\right|^{2} f\left(x, \rho^{2}\left|w^{\star}\right|^{2}\right)\right] d x \\
& +\frac{1}{2} \rho^{4} \int_{\mathbb{R}^{N}}\left\{(x \cdot \nabla W(x)) \star\left|w^{\star}\right|^{2}\right\}\left|w^{\star}\right|^{2} d x, \\
& I_{\omega}\left(u_{\rho}\right)=\frac{1}{2} \rho^{2} \int_{\mathbb{R}^{N}}\left\{2 \omega\left|w^{\star}\right|^{2}+\left|\nabla w^{\star}\right|^{2}\right. \\
& \left.+V(x)\left|w^{\star}\right|^{2}\right\} d x \\
& -\frac{1}{2} \int_{\mathbb{R}^{N}} F\left(x, \rho^{2}\left|w^{\star}\right|^{2}\right) d x \\
& -\frac{1}{4} \rho^{4} \int_{\mathbb{R}^{N}}\left(W(x) \star\left|w^{\star}\right|^{2}\right)\left|w^{\star}\right|^{2} d x .
\end{aligned}
$$

Since $f\left(x,\left|w^{*}\right|^{2}\right)<f\left(x, \rho^{2}\left|w^{*}\right|^{2}\right)$ and $\rho^{2} F\left(x,\left|w^{*}\right|^{2}\right)<$ $F\left(x, \rho^{2}\left|w^{*}\right|^{2}\right)$ for $\rho>1$ and from (38), we can obtain

$$
S_{\omega}\left(w_{\rho}\right)<\rho^{2} S_{\omega}\left(w^{\star}\right)=0, \quad Q\left(w_{\rho}\right)<\rho^{2} Q\left(w^{\star}\right)=0
$$

for any $\rho>1$. Noticing $d_{I I}>0$, we also can choose $\rho>1$ closing to 1 enough such that

$$
I_{\omega}\left(w_{\rho}\right)<\rho^{2} I_{\omega}\left(w^{\star}\right)<d_{I I}
$$

Equations (104) and (105) mean that $w_{\rho} \in \mathscr{K}$. That is, $\mathscr{K}$ is not empty.

Similar to (104), we can obtain

$$
S_{\omega}\left(w_{\rho}\right)>\rho^{2} S_{\omega}\left(w^{\star}\right)=0
$$

for any $0<\rho<1$. Noticing $d_{I I}>0$, we also can choose $0<\rho<1$ closing to 1 enough such that $I_{\omega}\left(w_{\rho}\right)<d_{I I}$ by continuity, which implies that $w_{\rho} \in \mathscr{R}_{+}$. That is, $\mathscr{R}_{+}$is not empty. 
For $w^{*} \in \sum$ satisfying $S_{\omega}\left(w^{*}\right)<0$ and $Q\left(w^{*}\right)=0$, letting $w_{\sigma}=\sigma w^{*}$ for $\sigma>0$, we have

$$
\begin{aligned}
Q\left(w_{\sigma}\right)= & \sigma^{2} \int_{\mathbb{R}^{N}}\left(2\left|\nabla w^{*}\right|^{2}-(x \cdot \nabla V(x))\left|w^{*}\right|^{2}\right) d x \\
& -\int_{\mathbb{R}^{N}} N\left[\sigma^{2}\left|w^{*}\right|^{2} f\left(x, \sigma^{2}\left|w^{*}\right|^{2}\right)\right. \\
& \left.-F\left(x, \sigma^{2}\left|w^{*}\right|^{2}\right)\right] d x \\
& +\frac{1}{2} \sigma^{4} \int_{\mathbb{R}^{N}}\left\{(x \cdot \nabla W(x)) \star\left|w^{*}\right|^{2}\right\}\left|w^{*}\right|^{2} d x, \\
S_{\omega}\left(w_{\sigma}\right)= & \sigma^{2} \int_{\mathbb{R}^{N}}\left\{2 \omega\left|w^{*}\right|^{2}+\left|\nabla w^{*}\right|^{2}+V(x)\left|w^{*}\right|^{2}\right\} d x \\
& -\int_{\mathbb{R}^{N}} \sigma^{2}\left|w^{*}\right|^{2} f\left(x, \sigma^{2}\left|w^{*}\right|^{2}\right) d x \\
& -\sigma^{4} \int_{\mathbb{R}^{N}}\left(W(x) \star\left|w^{*}\right|^{2}\right)\left|w^{*}\right|^{2} d x, \\
& \frac{1}{2} \sigma^{2} \int_{\mathbb{R}^{N}}\left\{2 \omega\left|w^{*}\right|^{2}+\left|\nabla w^{*}\right|^{2}+V(x)\left|w^{*}\right|^{2}\right\} d x \\
& -\frac{1}{2} \int_{\mathbb{R}^{N}} F\left(x, \sigma^{2}\left|w^{*}\right|^{2}\right) d x \\
& -\frac{1}{4} \sigma^{4} \int_{\mathbb{R}^{N}}\left(W(x) \star\left|w^{*}\right|^{2}\right)\left|w^{*}\right|^{2} d x .
\end{aligned}
$$

Since $\phi(\sigma)=Q\left(w_{\sigma}\right)$ is a smooth function of $\sigma$ and $Q\left(w^{*}\right)=0$, we have $\phi(1)=0$. If $\phi^{\prime}(1) \neq 0$, then there exists a $\sigma_{0}>0$ such that $Q\left(u_{\sigma}\right)=\phi(\sigma)>0$ for $\sigma \in\left(1, \sigma_{0}\right)$ if $\sigma_{0}>1$ (or $\sigma \in\left(\sigma_{0}, 1\right)$ if $\sigma_{0}<1$ ). By continuity, we can choose such $\sigma_{0}$ closing to 1 enough such that $S_{\omega}\left(w_{\sigma}\right)<0$ and $I_{\omega}\left(w_{\sigma}\right)<d_{I I}$ for $\sigma \in\left(1, \sigma_{0}\right)$ if $\sigma_{0}>1$ (or $\sigma \in\left(\sigma_{0}, 1\right)$ if $\left.\sigma_{0}<1\right)$. That is, $w_{\sigma} \in \mathscr{K}_{+}$and $\mathscr{K}_{+}$is not empty.

If $\phi^{\prime}(1)=0$, from $\phi(1)=0$ and $\phi^{\prime}(1)=0$, we can, respectively, obtain

$$
\begin{gathered}
-N \int_{\mathbb{R}^{N}}\left[\left|w^{*}\right|^{2} f\left(x,\left|w^{*}\right|^{2}\right)-F\left(x,\left|w^{*}\right|^{2}\right)\right] d x \\
=-N \int_{\mathbb{R}^{N}}\left|w^{*}\right|^{4} f_{s}^{\prime}\left(x,\left|w^{*}\right|^{2}\right) d x \\
+\frac{1}{2} \int_{\mathbb{R}^{N}}\left\{(x \cdot \nabla W(x)) \star\left|w^{*}\right|^{2}\right\}\left|w^{*}\right|^{2} d x \\
Q\left(w^{*}\right)=\int_{\mathbb{R}^{N}}\left(2\left|\nabla w^{*}\right|^{2}-(x \cdot \nabla V(x))\left|w^{*}\right|^{2}\right. \\
\left.\quad-N\left|w^{*}\right|^{4} f_{s}^{\prime}\left(x,\left|w^{*}\right|^{2}\right)\right) d x \\
+\int_{\mathbb{R}^{N}}\left\{(x \cdot \nabla W(x)) \star\left|w^{*}\right|^{2}\right\}\left|w^{*}\right|^{2} d x .
\end{gathered}
$$

Letting $w_{\sigma}=\sigma w^{*}$, we have

$$
\begin{aligned}
Q\left(w_{\sigma}\right)= & \sigma^{2} \int_{\mathbb{R}^{N}}\left(2\left|\nabla w^{*}\right|^{2}-(x \cdot \nabla V(x))\left|w^{*}\right|^{2}\right. \\
& \left.-N\left|w^{*}\right|^{4} f_{s}^{\prime}\left(x, \sigma^{2}\left|w^{*}\right|^{2}\right)\right) d x \\
& +\sigma^{4} \int_{\mathbb{R}^{N}}\left\{(x \cdot \nabla W(x)) \star\left|w^{*}\right|^{2}\right\}\left|w^{*}\right|^{2} d x \\
> & \sigma^{2} \int_{\mathbb{R}^{N}}\left(2\left|\nabla w^{*}\right|^{2}-(x \cdot \nabla V(x))\left|w^{*}\right|^{2}\right. \\
& +\sigma^{4} \int_{\mathbb{R}^{N}}\left\{(x \cdot \nabla W(x)) \star\left|w^{*}\right|^{2}\right\}\left|w^{*}\right|^{2} d x \\
= & \sigma^{2} Q\left(w^{*}\right)+\left(\sigma^{4}-\sigma^{2}\right) \\
& \times \int_{\mathbb{R}^{N}}\left\{(x \cdot \nabla W(x)) \star\left|w^{*}\right|^{2}\right\}\left|w^{*}\right|^{2} d x>0
\end{aligned}
$$

for $0<\sigma<1$. By continuity, we can choose such $\sigma$ closing to 1 enough such that $S_{\omega}\left(w_{\sigma}\right)<0$ and $I_{\omega}\left(w_{\sigma}\right)<d_{I I}$. That is to say, $w_{\sigma} \in \mathscr{K}_{+}$and $\mathscr{K}_{+}$is not empty.

(ii) In order to prove that $\mathscr{K}$ is the invariant manifold of (1), we need to show that, if $u_{0} \in \mathscr{K}$, then solution $u(x, t)$ of (1) satisfies $u(x, t) \in \mathscr{K}$ for any $t \in[0, T)$.

Assume that $u(x, t)$ is a solution of (1) with $u_{0} \in \mathscr{K}$. Then we can obtain

$$
\begin{aligned}
I_{\omega}(u(\cdot, t)) & =E(u(\cdot, t))+\omega\|u(\cdot, t)\|_{2}^{2} \\
& =E\left(u_{0}\right)+\omega\left\|u_{0}\right\|_{2}^{2}=I_{\omega}\left(u_{0}\right)<d_{I I}
\end{aligned}
$$

for $t \in[0, T)$. Next we prove that $S_{\omega}(u(\cdot, t))<0$ for $t \in[0, T)$. Otherwise, by continuity, there exists a $t_{0} \in(0, T)$ such that $S_{\omega}\left(u\left(\cdot, t_{0}\right)\right)=0$ because of $S_{\omega}\left(u_{0}\right)<0$. Since $\|u(\cdot, t)\|_{2}^{2}=\left\|u_{0}\right\|_{2}^{2}$ and $u_{0} \in \Sigma \backslash\{0\}$, it is easy to see that $u\left(\cdot, t_{0}\right) \in \Sigma \backslash\{0\}$. By the definitions of $d_{\mathcal{N}}$ and $d_{I I}$, we know that $I_{\omega}\left(u\left(\cdot, t_{0}\right)\right) \geq d_{\mathcal{N}} \geq$ $d_{I I}$, which is a contradiction to $I_{\omega}(u(\cdot, t))<d_{I I}$ for $t \in[0, T)$. Hence $S_{\omega}(u(\cdot, t))<0$ for all $t \in[0, T)$.

Now we only need to prove that $Q(u(\cdot, t))<0$ for $t \in$ $[0, T)$. Otherwise, since $Q\left(u_{0}\right)<0$, there exists a $t_{1} \in(0, T)$ such that $Q\left(u\left(\cdot, t_{1}\right)\right)=0$ by continuity. $S_{\omega}\left(u\left(\cdot, t_{1}\right)\right)<0$ means that $u\left(\cdot, t_{1}\right) \in \mathscr{C} \mathscr{M}$. By the definitions of $d_{\mathscr{M}}$ and $d_{I I}$, we obtain $I_{\omega}\left(u\left(\cdot, t_{1}\right)\right) \geq d_{M} \geq d_{I I}$, which is a contradiction to $I_{\omega}(u(\cdot, t))<d_{I I}$ for $t \in[0, T)$. Hence $Q(u(\cdot, t))<0$ for all $t \in[0, T)$.

By the discussions above, we know that $u(x, t) \in \mathscr{K}$ for any $t \in[0, T)$ if $u_{0} \in \mathscr{K}$, which means that $\mathscr{K}$ is the invariant manifold of (1).

Similarly, we can prove that $\mathscr{K}_{+}$, and $\mathscr{R}_{+}$are also invariant manifolds of (1).

Remark 27. By the definitions of $d_{I I}, d_{\mathcal{N}}, d_{\mathscr{M}}, \mathscr{K}, \mathscr{K}_{+}$, and $\mathscr{R}_{+}$, it is easy to see that

$$
\left\{u \in \Sigma \backslash\{0\}: I_{\omega}(u)<d_{I I}\right\}=\mathscr{K} \cup \mathscr{K}_{+} \cup \mathscr{R}_{+} .
$$


5.2. Proof of Theorem 7. Proof of Theorem 7 depends on the following two lemmas.

Lemma 28. Assume that the conditions of Theorem 7 hold. Then the solutions of (1) with $u_{0} \in \mathscr{K}$ will blow up in finite time.

Proof. Since $u_{0} \in \mathscr{K}$ and $\mathscr{K}$ is the invariant manifold of (1), we have $Q(u(x, t))<0, S_{\omega}(u(x, t))<0$, and $I_{\omega}(u(x, t))<d_{I I}$.

Under the conditions of Theorem 7 , we have $J^{\prime \prime}(t)=$ $4 Q(u)<0$ and $J^{\prime}(0)<0$. By the results of Proposition 10, the solution $u(x, t)$ will blow up in finite time. The conclusion of this lemma is true.

On the other hand, we have a parallel result on global existence.

Lemma 29. Assume that the conditions of Theorem 7 hold. If $u_{0} \in \mathscr{K}_{+}$or $u_{0} \in \mathscr{R}_{+}$, then the solutions of (1) exist globally.

Proof. Case 1. Assume that $u(x, t)$ is a solution of (1) with $u_{0} \in \mathscr{K}_{+}$. Since $\mathscr{K}_{+}$is an invariant manifold of (1), we know that $u(\cdot, t) \in \mathscr{K}_{+}$, which means that $I_{\omega}(u(\cdot, t))<d_{I I}$ and $Q(u(\cdot, t))>0 . Q(u(\cdot, t))>0$ and (23) imply that

$$
\begin{aligned}
2 \int_{\mathbb{R}^{N}}|\nabla u|^{2} d x-\int_{\mathbb{R}^{N}}(x \cdot \nabla V(x))|u|^{2} d x \\
\geq N l \int_{\mathbb{R}^{N}} F\left(x,|u|^{2}\right) d x \\
\quad-\frac{1}{2} \int_{\mathbb{R}^{N}}\left\{(x \cdot \nabla W(x)) \star|u|^{2}\right\}|u|^{2} d x .
\end{aligned}
$$

By the definition of $I_{\omega}(u)$ and using (113), we have

$$
\begin{aligned}
d_{I I}> & I_{\omega}(u(\cdot, t))=\omega \int_{\mathbb{R}^{N}}|u|^{2} d x \\
& +\frac{1}{2} \int_{\mathbb{R}^{N}}\left[|\nabla u|^{2}+V(x)|u|^{2}\right] d x \\
& -\frac{1}{2} \int_{\mathbb{R}^{N}} F\left(x,|u|^{2}\right) d x \\
& -\frac{1}{4} \int_{\mathbb{R}^{N}}\left(W(x) \star|u|^{2}\right)|u|^{2} d x \\
\geq & \omega \int_{\mathbb{R}^{N}}|u|^{2} d x+\frac{N l-2}{2 N l} \int_{\mathbb{R}^{N}}|\nabla u|^{2} d x \\
& +\int_{\mathbb{R}^{N}} \frac{N l V(x)+(x \cdot \nabla V(x))}{2 N l}|u|^{2} d x \\
& -\frac{1}{4 N l} \int_{\mathbb{R}^{N}}\{[N l W(x)+(x \cdot \nabla W(x))] \\
\geq & C\left(\int_{\mathbb{R}^{N}}|u|^{2} d x+\int_{\mathbb{R}^{N}}|\nabla u|^{2} d x+\int_{\mathbb{R}^{N}} V(x)|u|^{2} d x\right) .
\end{aligned}
$$

Equation (114) means that $u(x, t)$ exists globally.
Case 2. Assume that $u(x, t)$ is a solution of (1) with $u_{0} \in$ $\mathscr{R}_{+}$. Since $\mathscr{R}_{+}$is also an invariant manifold of (1), we know that $u(x, t), \in \mathscr{R}_{+}$, which means that $I_{\omega}(u(\cdot, t))<d_{I I}$ and $S_{\omega}(u(\cdot, t))>0$. Since $S_{\omega}(u)>0$, we can get

$$
\begin{aligned}
\omega\|u\|_{2}^{2} & +\frac{1}{2} \int_{\mathbb{R}^{N}}\left(|\nabla u|^{2}+V(x)|u|^{2}\right) d x \\
> & \frac{1}{2} \int_{\mathbb{R}^{N}} f\left(x,|u|^{2}\right)|u|^{2} d x \\
& +\frac{1}{2} \int_{\mathbb{R}^{N}}\left(W(x) \star|u|^{2}\right)|u|^{2} d x \\
\geq & \min (l+1,2)\left(\frac{1}{2} \int_{\mathbb{R}^{N}} F\left(x,|u|^{2}\right) d x\right. \\
& \left.+\frac{1}{4} \int_{\mathbb{R}^{N}}\left(W(x) \star|u|^{2}\right)|u|^{2} d x\right) .
\end{aligned}
$$

From (115), we can obtain

$$
\begin{aligned}
I_{\omega}(u)= & \omega\|u\|_{2}^{2}+\frac{1}{2} \int_{\mathbb{R}^{N}}\left[|\nabla u|^{2}+V(x)|u|^{2}\right. \\
& \left.-F\left(x,|u|^{2}\right)\right] d x-G\left(|u|^{2}\right) \\
\geq & \min \left(\frac{l}{(l+1)}, \frac{1}{2}\right) \\
& \times\left(\omega\|u\|_{2}^{2}+\frac{1}{2} \int_{\mathbb{R}^{N}}\left[|\nabla u|^{2}+V(x)|u|^{2}\right] d x\right) .
\end{aligned}
$$

Equation (116) implies that the solution $u(x, t)$ exists globally.

Proof of Theorem 7. By the results of Lemmas 28 and 29, we know that Theorem 7 is right.

As a corollary of Theorem 7, we obtain a sharp threshold for the blowup in finite time and global existence of the solution of (9) as follows.

Corollary 30. Assume that $f\left(x,|u|^{2}\right) \equiv 0, V(x) \equiv 0, W(x)>$ 0 for all $x \in \mathbb{R}^{N}, W(x)$ is even, and $W(x) \in L^{\infty}\left(\mathbb{R}^{N}\right)+$ $L^{q}\left(\mathbb{R}^{N}\right)$ with some $q>N / 4$. Suppose further that there exists $l$ satisfying $2<N l$ and

$$
N l W(x)+(x \cdot \nabla W(x)) \leq 0 .
$$

If $u_{0} \in H^{1}\left(\mathbb{R}^{N}\right),|x| u_{0} \in L^{2}\left(\mathbb{R}^{N}\right)$, and $I_{\omega}\left(u_{0}\right)=\omega\left\|u_{0}\right\|_{2}^{2}+$ $E\left(u_{0}\right)<d_{I I}$, then the solution of (9) blows up in finite time if and only if $u_{0} \in \mathscr{K}$.

Remark 31. A typical example is

$$
\begin{gathered}
-i u_{t}=\Delta u+\left(|x|^{-K} \star|u|^{2}\right) u, \quad x \in \mathbb{R}^{N}, t>0, \\
u(x, 0)=u_{0}(x), \quad x \in \mathbb{R}^{N},
\end{gathered}
$$


which is also a special case of (1) with $V(x) \equiv 0, f\left(x,|u|^{2}\right) \equiv 0$, and $W(x)=|x|^{-K}$ with $2<N l<K<N / q<4$. Letting $W(x)=W_{1}(x)+W_{2}(x)$ with

$$
\begin{aligned}
& W_{1}(x)= \begin{cases}0, & |x| \leq 1, \\
|x|^{-K}, & |x|>1,\end{cases} \\
& W_{2}(x)= \begin{cases}|x|^{-K}, & |x| \leq 1, \\
0, & |x|>1,\end{cases}
\end{aligned}
$$

we can see that $W_{1}(x) \in L^{\infty}\left(\mathbb{R}^{N}\right)$ and $W_{2}(x) \in L^{q}\left(\mathbb{R}^{N}\right)$ with some $N / 4<q<N / 2$. Corollary 30 gives the sharp threshold for blowup and global existence of the solution to (118).

\section{Acknowledgments}

The authors are grateful to the referees for their helpful comments. In addition, the second author is supported by the National Natural Science Foundation of China, Grant 11071185 and Natural Science Foundation of Tianjin (09JCYBJC01800). The third author is supported by the National Natural Science Foundation of China, Grant 11071237.

\section{References}

[1] T. Cazenave, Semilinear Schrödinger Equations, vol. 10, American Mathematical Society, Providence, RI, USA, 2003.

[2] Y.-G. Oh, "Cauchy problem and Ehrenfest's law of nonlinear Schrödinger equations with potentials," Journal of Differential Equations, vol. 81, no. 2, pp. 255-274, 1989.

[3] Z. Gan, B. Guo, and J. Zhang, "Blowup and global existence of the nonlinear Schrödinger equations with multiple potentials," Communications on Pure and Applied Analysis, vol. 8, no. 4, pp. 1303-1312, 2009.

[4] Z. Gan and J. Zhang, "Sharp threshold of global existence and instability of standing wave for a Davey-Stewartson system," Communications in Mathematical Physics, vol. 283, no. 1, pp. 93125, 2008.

[5] J. Zhang, "Sharp threshold for blowup and global existence in nonlinear Schrödinger equations under a harmonic potential," Communications in Partial Differential Equations, vol. 30, no. 10, pp. 1429-1443, 2005.

[6] R. T. Glassey, "On the blowing up of solutions to the Cauchy problem for nonlinear Schrödinger equations," Journal of Mathematical Physics, vol. 18, no. 9, pp. 1794-1797, 1976.

[7] H. Berestycki and T. Cazenave, "Instabilite des etats stationnaires dans les equations de Schrödinger et de Klein-Gordon non lineaires," Comptes Rendus de l'Académie des Sciences, vol. 293, pp. 489-492, 1981.

[8] M. I. Weinstein, "Nonlinear Schrödinger equations and sharp interpolation estimates," Communications in Mathematical Physics, vol. 87, no. 4, pp. 567-576, 1983.

[9] T. Cazenave and F. Weissler, Some Remarks on the Nonlinear Schrödinger Equation in the Critical Case, Nonlinear Semigroups, Partial Dierential Equations and Attractors, Lecture Notes in Mathematics, Springer, New York, NY, USA, 1989, Edited by T. L. Gill and W. W. Zachary.

[10] T. Tao, M. Visan, and X. Zhang, "The nonlinear schrödinger equation with combined power-type nonlinearities," Communications in Partial Differential Equations, vol. 32, no. 8, pp. 12811343, 2007.
[11] G. Chen, J. Zhang, and Y. Wei, "Energy criterion of global existence for supercritical nonlinear Schrödinger equation with harmonic potential," Journal of Mathematical Physics, vol. 48, no. 7, Article ID 073513, 2007.

[12] G. G. Chen, J. Zhang, and Y. Y. Wei, "A small initial data criterion of global existence for the damped nonlinear Schrödinger equation," Journal of Physics, vol. 42, no. 5, p. 7, 2009.

[13] D. Li, C. Miao, and X. Zhang, "The focusing energy-critical Hartree equation," Journal of Differential Equations, vol. 246, no. 3, pp. 1139-1163, 2009.

[14] C. Miao, G. Xu, and L. Zhao, "Global well-posedness and scattering for the mass-critical Hartree equation with radial data," Journal des Mathematiques Pures et Appliquees, vol. 91, no. 1, pp. 49-79, 2009.

[15] C. X. Miao, G. X. Xu, and L. F. Zhao, "Global well-posedness, scattering and blow-up for the energy-critical, focusing Hartree equation in the radial case," Colloquium Mathematicum, vol.114, pp. 213-236, 2009.

[16] R. Carles, "Nonlinear Schrödinger equations with replusive harmonic potemtial and applications," SIAM Journal on Mathematical Analysis, vol. 35, pp. 823-843, 2003.

[17] P. H. Rabinowitz, "On a class of nonlinear Schrödinger equations," Zeitschrift Für Angewandte Mathematik Und Physik, vol. 43, pp. 270-291, 1992. 


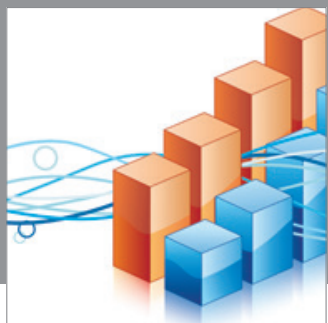

Advances in

Operations Research

mansans

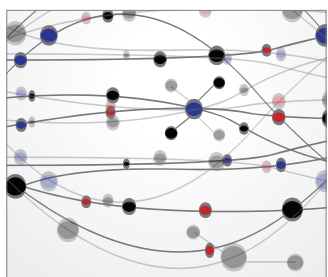

The Scientific World Journal
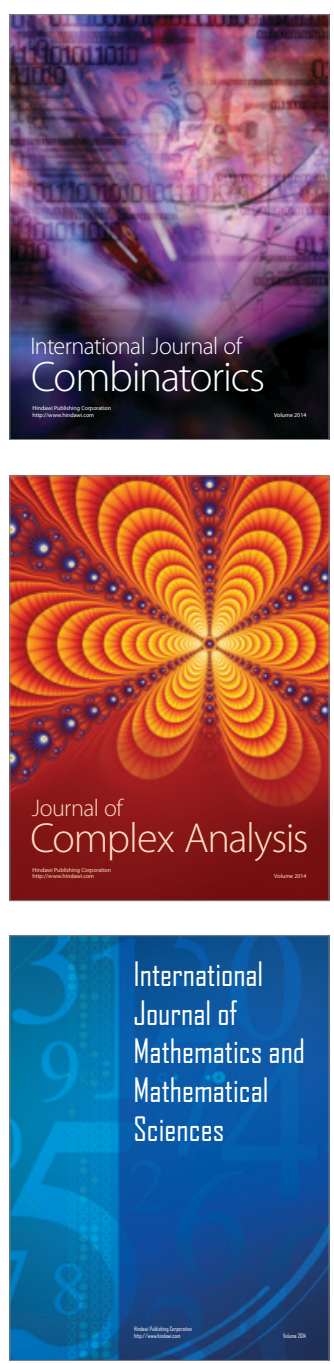
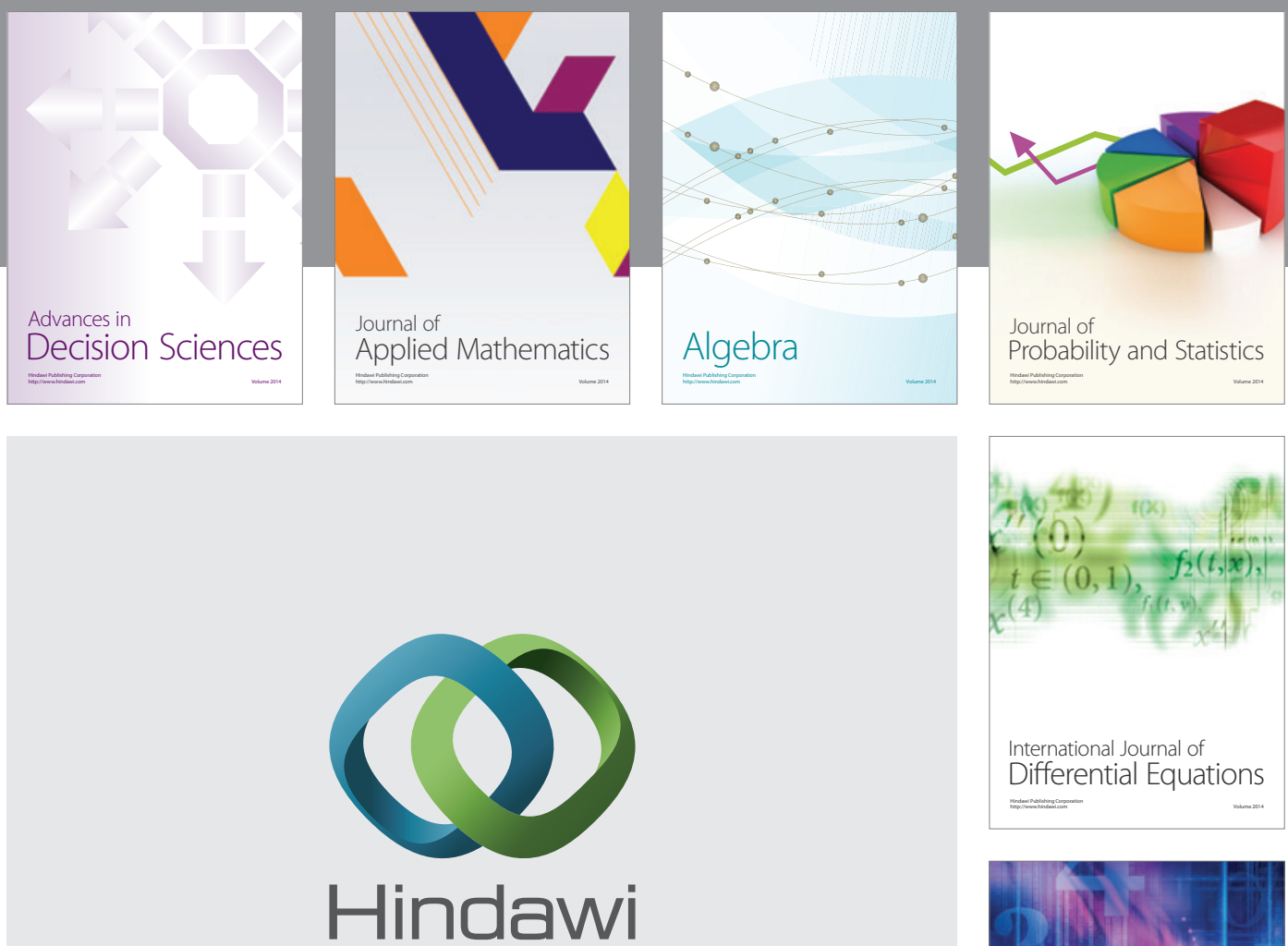

Submit your manuscripts at http://www.hindawi.com
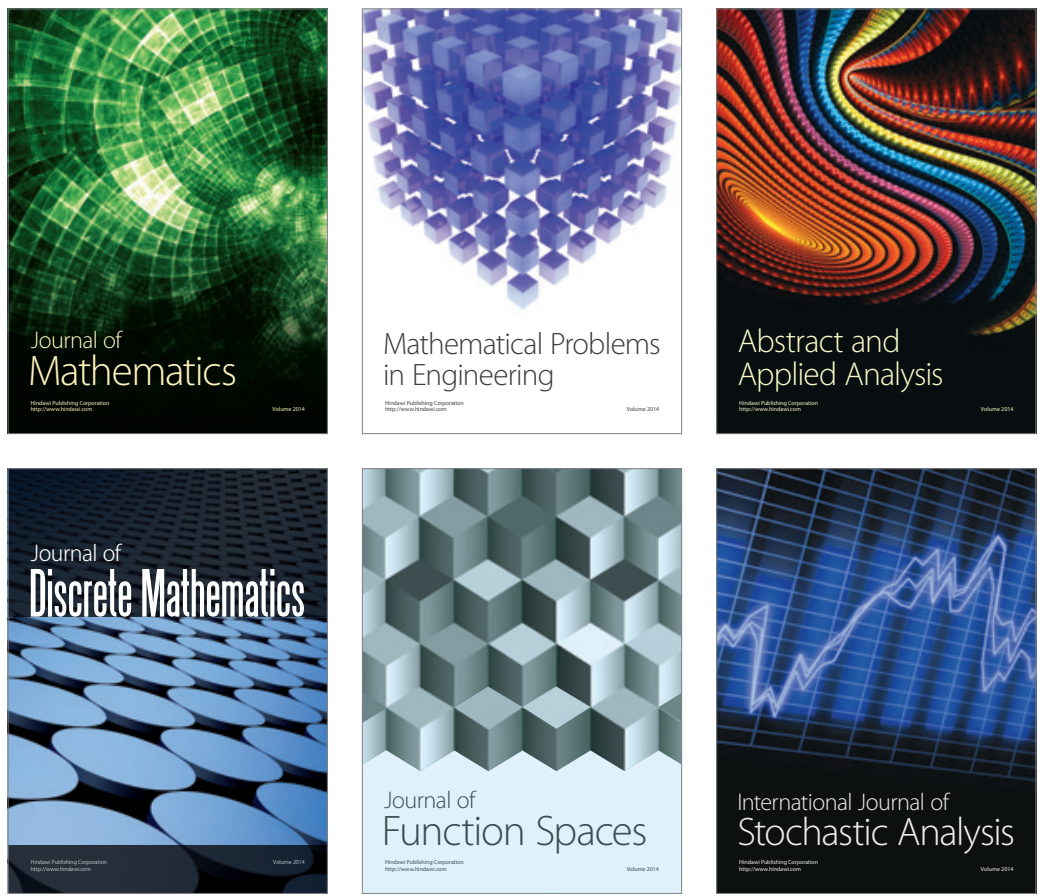

Journal of

Function Spaces

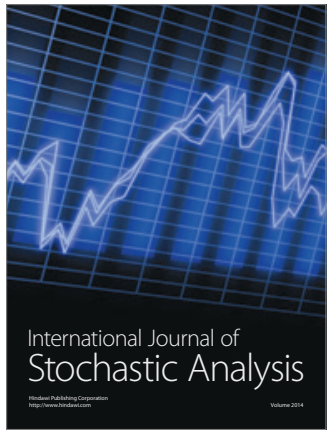

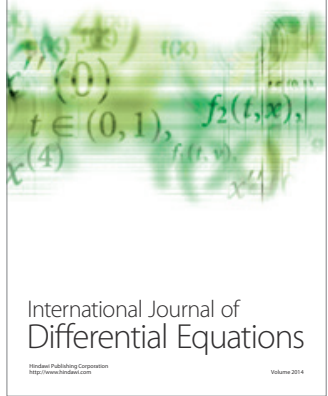
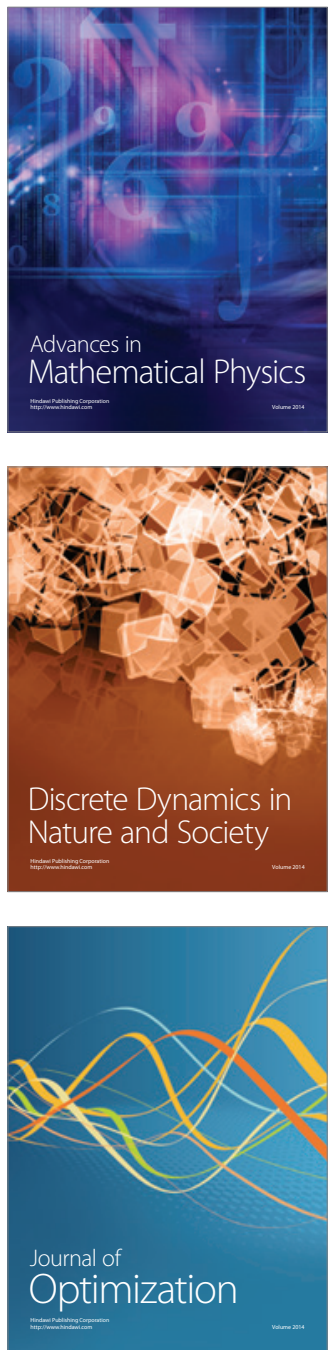